DESY M-91-06

May 1991

Wake Fields and Impedances

T. Weiland, R. Wanzenberg 


\title{
Wake Fields and Impedances
}

\author{
T. Weiland, R. Wanzenberg
}

\section{Contents}

1 Introduction 2

1.1 Basic Concepts ... . . . . . . . . . . . . . . 2

1.2 Some simple examples.................... 4

2 Wake Fields 6

2.1 Wake Fields in a Resonant Cavity with Beann Pipes . . . . . . . . . 6

2.2 Basic Definitions . . . . . . . . . . . . . . . . 8

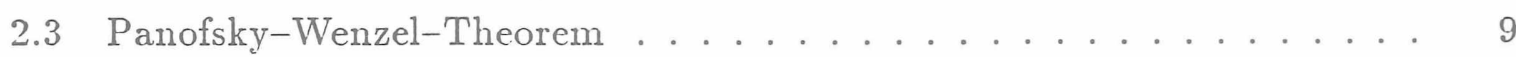

2.4 Wake Potential in Cylindrical Symmetric Structures . . . . . . . . 11

2.5 Fully 3 -D Structures ...................... 13

3 Impedances 16

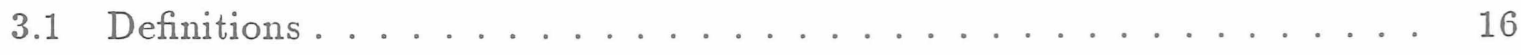

3.2 Loss Parameters . . . . . . . . . . . . . . . . . . . . . . 17

3.3 Fundamental Theorem of Beam Loading . . . . . . . . . . . . 21

3.4 Shunt Impedance and Quality Factor . . . . . . . . . . . . . . 22

4 Analytical and Numerical Calculations of Wake Fields 25

4.1 Analytical Calculation for a Pill Box . . . . . . . . . . . 25

4.2 Numerical Calculations . . . . . . . . . . . . . . . . . . 28

4.2.1 Grid Maxwell Equations . . . . . . . . . . . . . . . . . 28

4.2.2 Short and Long Range Wakes . . . . . . . . . . . . . . . 33

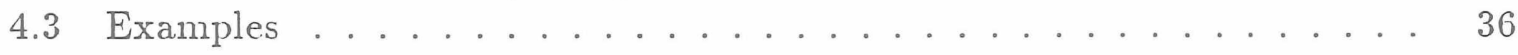

5 Effects of Wakes and Impedances 41

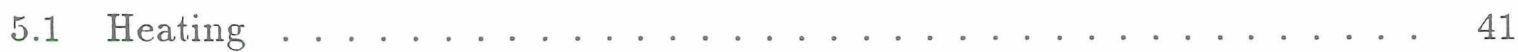

5.2 Instabilities . . . . . . . . . . . . . . . . . . 42

6 Strategies in Accelerator Design 45

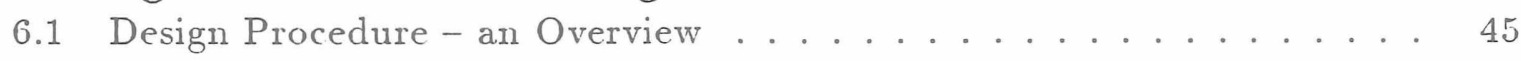

6.2 Parasitic Losses versus Shunt Impedance. . . . . . . . . . . . . . . . 46

$\begin{array}{ll}\text { Appendix A } & 48\end{array}$ 


\section{Introduction}

In this lecture the concepts of wake fields and impedances are introduced to describe the electromagnetic interaction of a bunch of charged particles in the accelerator with its enviromment. The various components of the enviromment are the vacuum chamber, cavities, bellows, dielectric coated pipes, and all other kind of obstacles the beam has to pass during his way through the accelerator.

Since the purpose of this paper is tutorial and not original nor exhausting material is freely taken from previous papers and reports when appropriate. The plan of the lecture is to present first some typical examples from accelerator physics where wake field effects are important. This material is presented in the-following subsections of this introduction.

In section 2, the concept of wake potential is introduced. Multipole expansions are studied for structures with cylindrical symmetry. The interdependence of longitudinal and transverse wake forces are examined in the light of the Panofsky-Wenzel-Theorem. Even for fully 3-D structures results of numerical calculations are presented.

In section 3 , the impedance is introduced as the fourier transform of the wake potential. The coupling of the beam to one mode of a cavity leads to the concept of the loss parameter. The fundamental theorem of beam loading is explained. The connection between the loss parameter and the shunt impedance is established.

In section 4, different methods actually calculating the wake potentials are introduced. Analytical calculations for a pill box cavity are presented. Numerical calculations in the time and frequency domain for arbitrarily shaped structures are shown. On the basis of examples it is demonstrated that mode summation methods are only appropriated for long range wake potential calculations while short range wakes are only properly calculated by computer codes working in the time domain. In order to avoid that the wake potential computations by codes like TBCI or MAFIA appears to be a magic black box the basic methods with Maxwell grid equations are explained.

The effects of wakes and impedances and their importance for accelerators of all types are explained in section 5. Since instabilities are an extensive subject this section can only be exemplary and not in the least exhaustive.

As a conclusion, in section 6, strategies in accelerator design are presented. It is demonstrated that increasing the shunt impedance of a cavity will also increase parasitic losses to higher order modes. Hence an increase of the shunt impedance can spoil the performance of the whole accelerator if higher currents should be used.

\subsection{Basic Concepts}

Consider a point charge $q$ moving in free space at a velocity $v$ close to the speed of light. The electromagnetic field carried by a relativistic point charge is treated in many text. books of electrodynamics (see for example [1]). The field distribution is shown in figure 1.1. The Electromagnetic field is Lorentz contracted into a thin disk perpendicular 


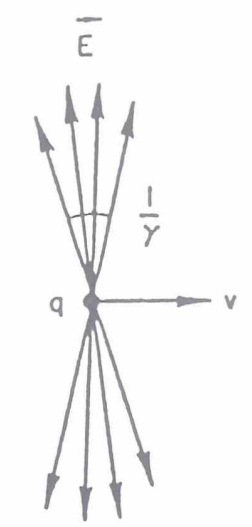

Figure 1: Electromagnetic field carried by a relativistic point charge

to the particles direction of motion, which we choose to be the $z$ - axis in a cylindrical coordinate system. The opening angle of the field distribution is on the order of $1 / \gamma$, where $\gamma=\left(1-(v / c)^{2}\right)^{-1 / 2}$.

In the ultrarelativistic limit $v \rightarrow c($ or $\gamma \rightarrow \infty)$ the disk containing the field shrinks into a $\delta$ - function distribution. The nonvanishing field components are:

$$
E_{r}=\frac{q}{2 \pi \varepsilon_{0} r} \delta(z-c t), \quad H_{\varphi}=\frac{E_{r}}{Z_{0}}, \quad \text { with } Z_{0}=377 \Omega
$$

Since the electric field $E$ points strictly radically outward from the point charge, all field components are identically zero both ahead and behind the point charge, and hence there are no forces on a test particle either preccding or following the charge $q$. This is a consequence of the principle of causality.

For $v$ slightly less then $c$ this is not strictly true. But. if we look at typical charges and energies of $\epsilon^{+} \epsilon^{-}$high energy accelerator:

\begin{tabular}{l|r|r|r}
\hline & charge & energy & $\gamma=\left(1-(v / c)^{2}\right)^{-1 / 2}$ \\
\hline PETRA & $40 \mathrm{nC}$ & $20 \mathrm{GeV}$ & 40000 \\
LEP & $50 \mathrm{nC}$ & $50 \mathrm{GeV}$ & 100000 \\
SLC & $2 \mathrm{nC}$ & $50 \mathrm{GeV}$ & 100000
\end{tabular}

and compare the space charge force $V_{s}=e /\left(4 \pi \epsilon_{0} d^{3} \gamma^{2}\right)$ (d the rms distant between two electrons in the bunch) with the accelerating force $V_{a c c}$ it is obvious that in a good approximation any space charge effects can be neglected, for sufficient long bunches. Due to the higher rest mass of protons the argument applies not to them for the same energy range. Hence for protons or even heavy ions it is necessary to practice more caution.

In the following section we will restrict us to the ultrarelativistic case $(\gamma=\infty, v=$ c). So space charge effects are neglected. 


\subsection{Some simple examples}

Consider now some typical situations where electromagnetic fields occure behind the point charge $q$ moving with velocity $c$ through a structure.

Let us start. with a charge moving through a cylindrical pipe on the $z$-axis. All electric field lines terminate transversely on surface charges on the wall of the pipe. If the pipe is perfectly conducting these image charges move in synchronism with the charge on the axis. Hence there will be no wake fields behind the charge. But the situation is somehow different, if the material in the walls has a nonvanishing resistivity. Figure 1.2 shows the resistive wall wake fields generated by a point charge $q$ traversing a pipe with materials of finite conductivity in the walls. For a Gaussian bunch $(\sigma=1 \mathrm{~mm}$

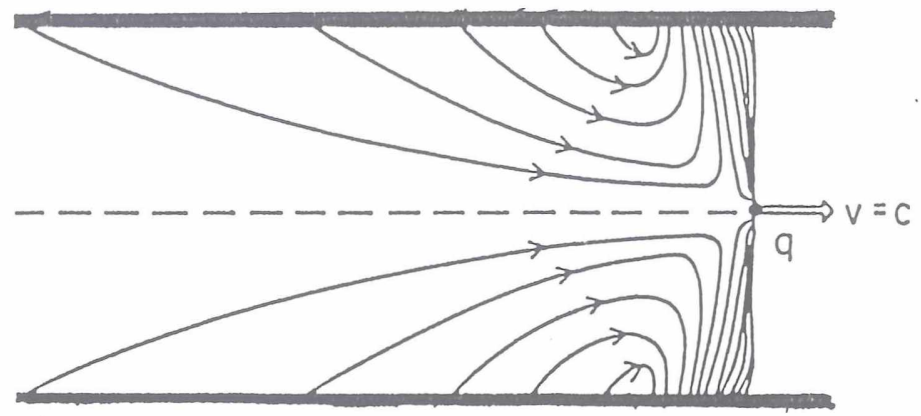

Figure 2: Resistive wall wake fields in a pipe with materials in the walls with finite conductivity.

rms bunch length) of a total charge of $10 \mathrm{nC}$ traversing a copper pipe with radius $1 \mathrm{~cm}$ the typical maximal wake field is in the order of magnitude of $1 \mathrm{kV} / \mathrm{m}[3]$.

The next example is a dielectric coated pipe. Such kind of structures could be used as travelling wave acceleration sections. The phase velocity of the wave is below the velocity of light due to the dielectric coating. At a first glance one might think that the wake fields are significantly smaller than in commonly iris loaded structures. But calculations show [2] that this is not the case. For a charge of $10 \mathrm{nC}$ typic al wake fields of about $500 \mathrm{kV} / \mathrm{m}$ where found. The inner pipe radius was $1 \mathrm{~cm}$, the coating thickness $3 \mathrm{~cm}$ with a dielectric constant of $2 \times \varepsilon_{0}$. Another problem is the bad thermal conductivity of the dielectric coating, which lead to severe heating problems due to electric losses.

The last example of the introductory section is the important case of an obstacle in a beam pipe. Figure 1.2 shows the electric field lines for fire successive time steps with the computer code TBCI. For a gaussian charge distribution (rms length $\sigma=1$ $\mathrm{cm}$ ) with total charge of $10 \mathrm{nC}$ the wake fields behind the bunch are about $20 \mathrm{MV} / \mathrm{m}$. The pipe has radius of $1 \mathrm{~cm}$, and the iris radius is $0.2 \mathrm{~mm}$. 
From this basic considerations we have learned that for electron accclerators the dominant forces are caused by geometrical changes along the beam pipe. Space charge effects are negligible for ultrarelativistic particles. Resistive wall wakes are in most cases negligible. Dielectric coated structures should always checked in detail for the specific application.
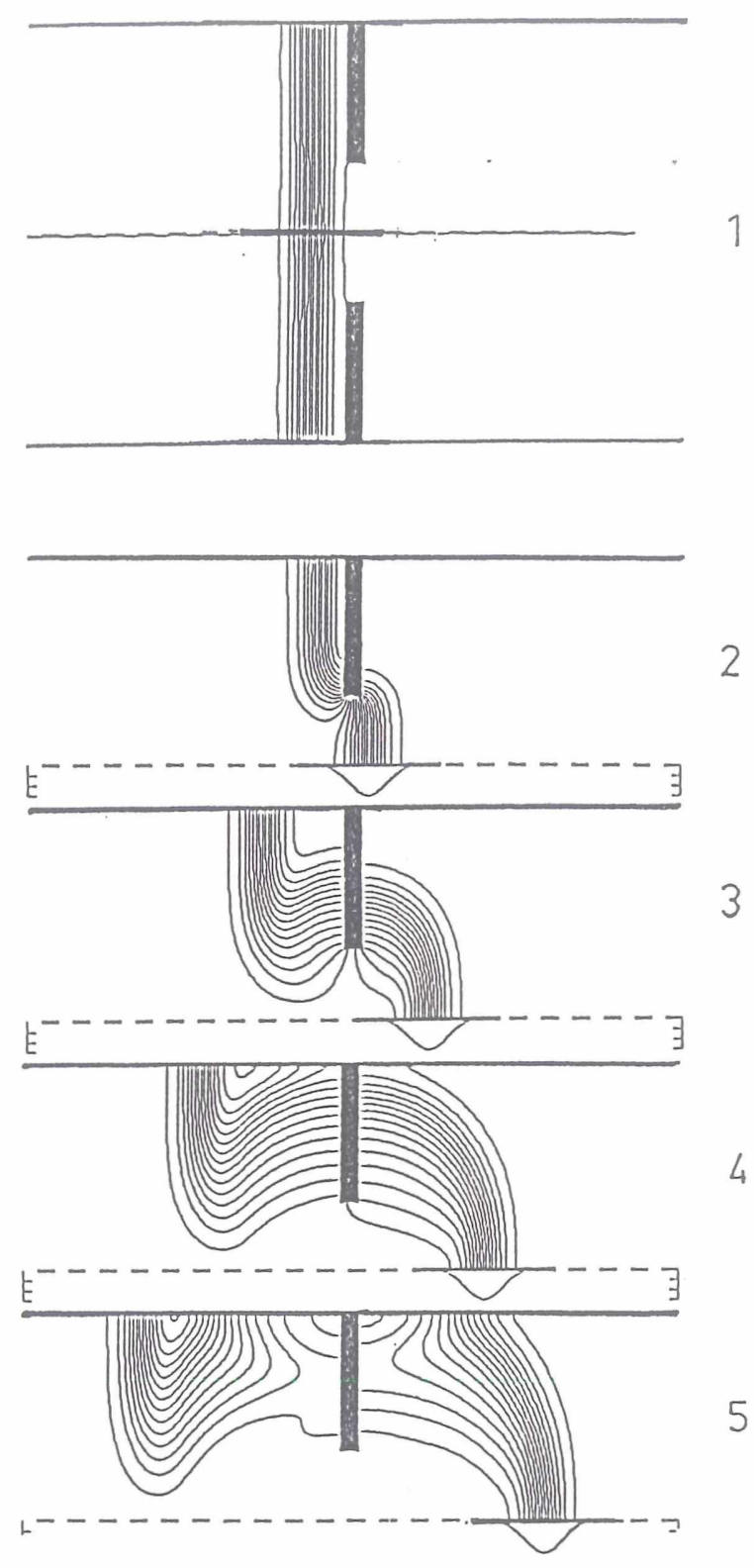

Figure 3: Wake fields generated by a Gaussian charge distribution passing an obstacle in the beam pipe. 


\section{Wake Fields}

\subsection{Wake Fields in a Resonant Cavity with Beam Pipes}

The examples of the introduction provide a qualitative understanding about what wake fields are, and how they are generated. Before we proceed to some mathematical describtions in terms of wake potentials consider another example. An ultrarelativistic bunch with total charge $q_{1}$ transverse a small cavity along the $z$ - axis (see figure 2.1).

The electromagnetic force on any test charge $q_{2}$ is given by the Lorentz equation as a. function of space and time coordinates:

$$
\boldsymbol{F}(\boldsymbol{r}, t)=q_{2}(\boldsymbol{E}(\boldsymbol{r}, t)+\boldsymbol{v} \times \boldsymbol{B}(\boldsymbol{r}, t))
$$

The fields $E$ and $B$ are generated by the Gaussian charge distribution $\rho$. They are solutions of the Maxwell equations and have to obey several boundary conditions.

$$
\begin{array}{ll}
\nabla \times B=\mu_{0} \boldsymbol{j}+\frac{1}{c^{2}} \frac{\partial}{\partial t} \boldsymbol{E} & \nabla \cdot \boldsymbol{B}=0 \\
\nabla \times \boldsymbol{E}=-\frac{\partial}{\partial t} \boldsymbol{B} & \nabla \cdot \boldsymbol{E}=\frac{1}{\epsilon_{0}} \rho .
\end{array}
$$

In our case the charge distribution is:

$$
\rho(\boldsymbol{r}, t)=q_{1} \lambda_{\perp}(x, y) \lambda(z-c t), \quad \lambda(s)=\frac{1}{\sigma \sqrt{2 \pi}} \exp \left(-\frac{\left(s-s_{0}\right)^{2}}{2 \sigma^{2}}\right) .
$$

$\lambda_{\perp}$ is the transverse distribution. $\lambda$ is the longitudinal Gaussian distribution (centered around $s_{0}$ ). The corresponding current density is given by

$$
j(r, t)=c e_{z} \rho(\boldsymbol{r}, t) .
$$

A numerical calculation of the electrical fields lines for three subsequent time steps is shown in figure 2.1. After the interaction of the bunch with the cavity there remain electromagnetic fields in the cavity. The bunch has lost energy into one or in general several cavity modes. Consider now a test charge $q_{2}$ following the bunch in a distant $s$ with the same velocity $v \approx c$. The Lorentz force is:

$$
\boldsymbol{F}(s, t)=q_{2}\left(\boldsymbol{E}(z=c t-s, t)+c \boldsymbol{e}_{z} \times \boldsymbol{B}(z=c t-s, t)\right)
$$

We can distinguish between two regimes, the transient regime due to short range wake effects and the resonant regime due to long range wake effects. For small distances $s$ (inside the bunch) transient wake field effects are dominant while for $s \gg \sigma$ ( $\sigma$ the bunch length) the wake field is dominated by resonant effects due to several cavity modes ringing with their specific frequencies.

The net momentum change of the test charge can be calculated as an average over the Lorentz force:

$$
\Delta p \sim \int d \cdot F(s, t)
$$

This leads to the concept of wake potentials. 

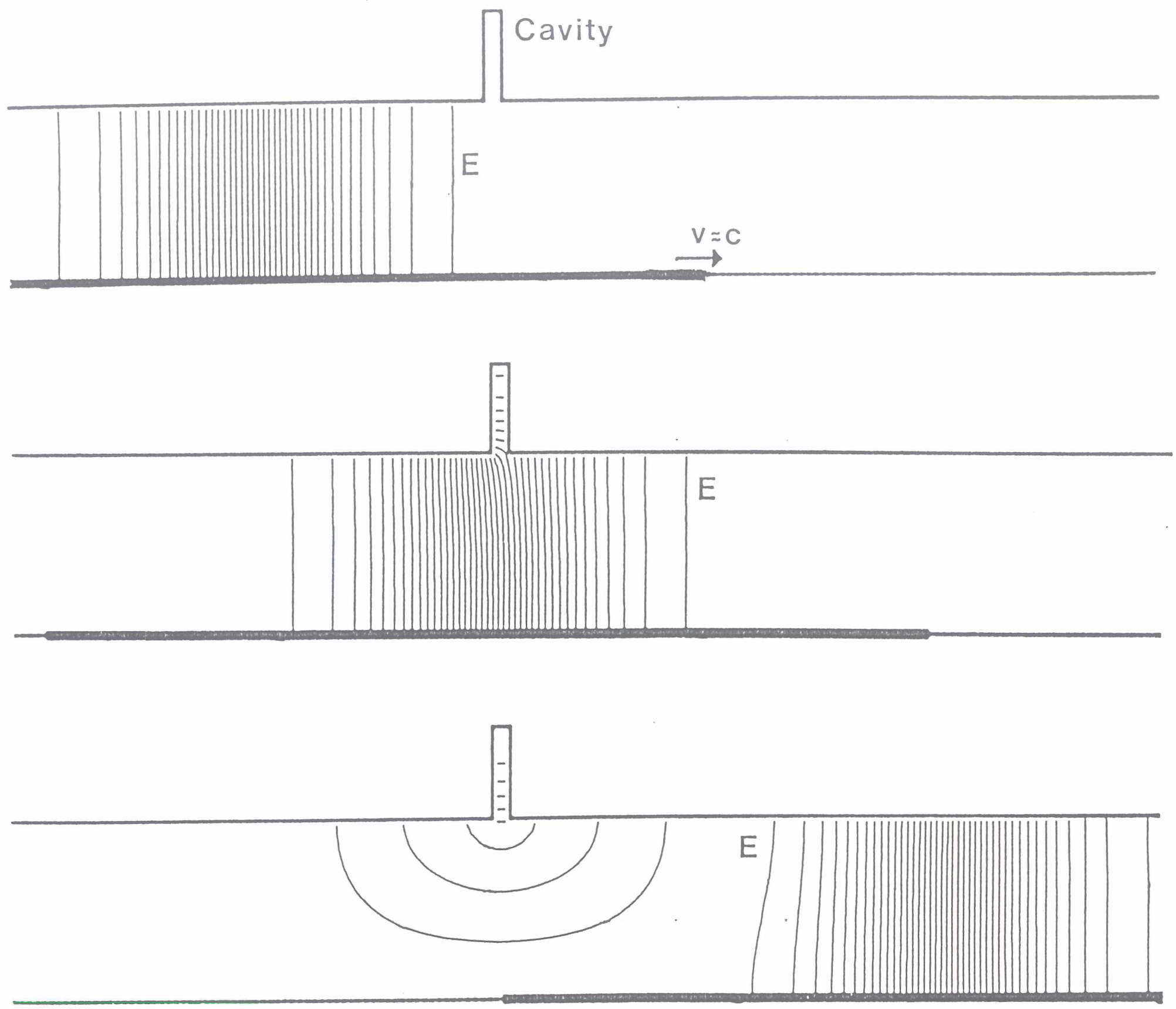

Figure 4: Wake fields generated by a Gaussian bunch transversing a cavity. The electric field is represented by lines of constant $\int d t r H_{\varphi} \sim r|E|$ 


\subsection{Basic Definitions}

Consider a charge distribution with total charge $q_{1}$ traversing a structure with offset $r_{1}$ parallel to the $z$-axis with the speed of light (see figure 5 ). Then the Wake Potential is defined as:

$$
\boldsymbol{W}\left(r_{1}, s\right)=\frac{1}{q_{1}} \int_{-\infty}^{\infty} d z\left[\boldsymbol{E}\left(r_{1}, z, t\right)+c \boldsymbol{e}_{z} \times \boldsymbol{B}\left(r_{1}, z, t\right)\right]_{t=(s+z) / c} .
$$

The distant $s$ is measured from the head of the bunch $q_{1}$ into the opposite direction of $v$ The change of momentum of a test charge $q_{2}$ following behind the bunch at a distant $s$ on the same path is given by:

$$
\Delta \boldsymbol{p}=q_{1} q_{2} \boldsymbol{W}(s)
$$

Since $e_{z} \cdot\left(e_{z} \times B\right)=0$ the longitudinal component of the wake potential is simply

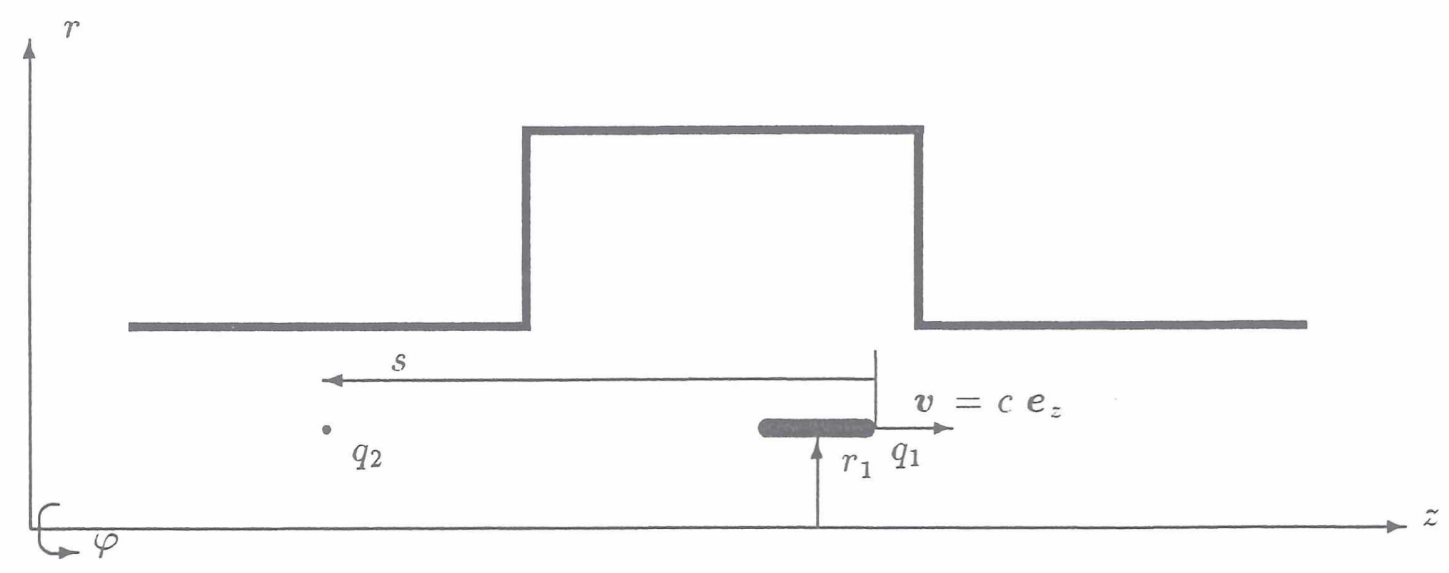

Figure 5: A bunch with total charge $q_{1}$ traverse a cavity followed by a test charge $q_{2}$.

given by:

$$
W_{\|}\left(r_{1}, s\right)=\frac{1}{q_{1}} \int_{-\infty}^{\infty} d z E_{z}\left(r_{1}, z,(s+z) / c\right) .
$$

In figure 2.2 the normalized longitudinal component of the wake potential for the above considered example with the small cavity is shown. The dotted line represents the Gaussian charge distribution in the range from $-5 \sigma$ to $5 \sigma$. Due to the transient. wake field effects the head of the bunch (left hand side of the figure) is decelerated. While a test charge at a certain position behind the bunch will be accelerated. 


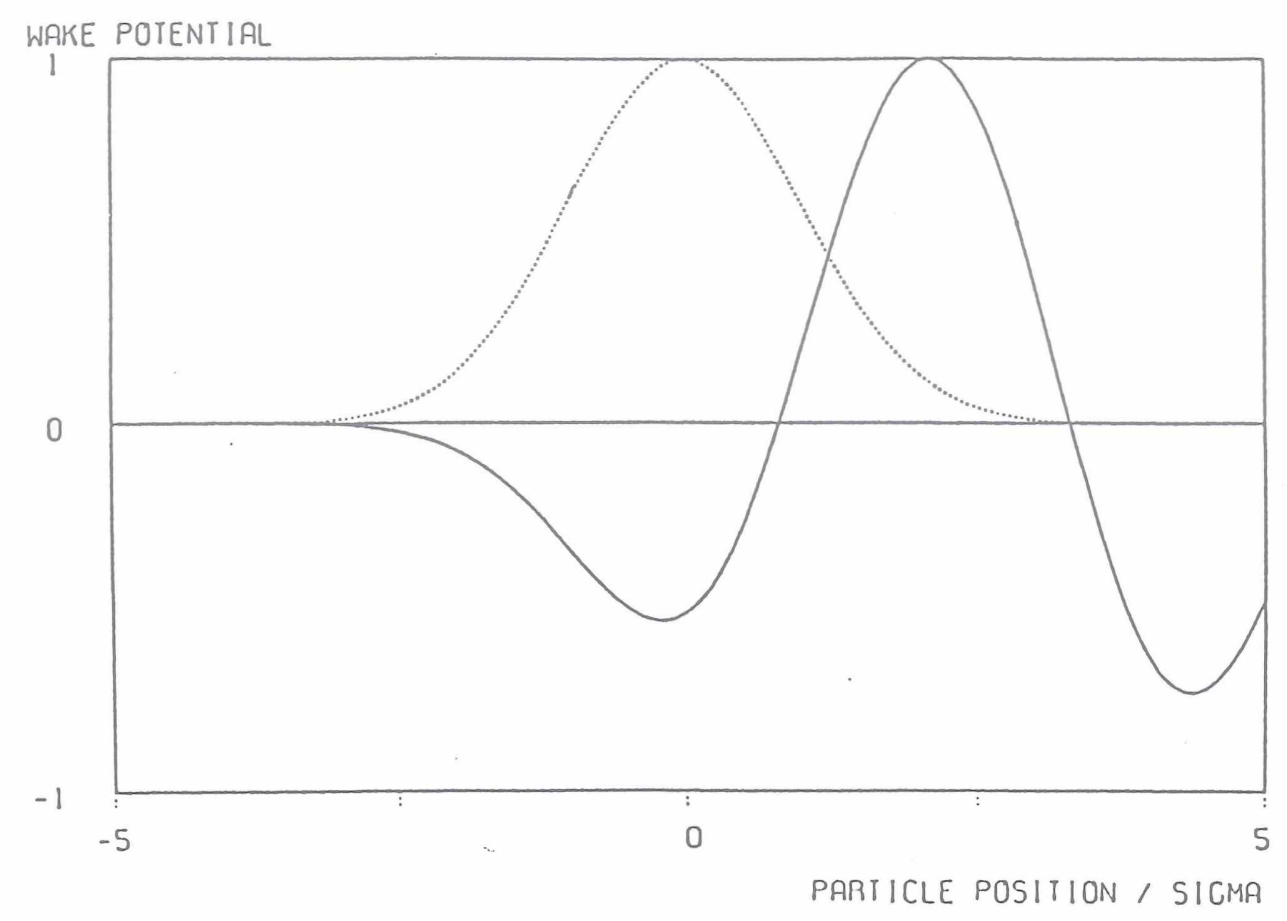

Figure 6: Longitudinal Wake Potential

\subsection{Panofsky-Wenzel-Theorem}

In principle it is sufficient to know the longitudinal wake potential for all transverse positions $(x, y)$ or $(r, \varphi)$ because it is possible to reconstruct the transverse component. of the wake potential.

From the Maxwell equation $\nabla \times \boldsymbol{E}=-\frac{\partial}{\partial t} B$ we obtain:

$$
e_{z} \times \frac{\partial}{\partial t} B=\frac{\partial}{\partial z} E_{\perp}-\nabla_{\perp} E_{z}
$$

Since the total derivative of the transverse component of the electric field with respect to $z$ is given by

$$
\frac{d}{d z} \boldsymbol{E}_{\perp}\left(x, y, z, \frac{z+s}{c}\right)=\left(\frac{\partial}{\partial z}+\frac{1}{c} \frac{\partial}{\partial t}\right) \boldsymbol{E}_{\perp}\left(x, y, z, \frac{z+s}{c}\right),
$$

the derivative of the transvers wake potential with respect to s can be written as:

$$
\frac{\partial}{\partial s} \boldsymbol{W}_{\perp}(x, y, s)=\frac{1}{q_{1}} \int_{-\infty}^{\infty} d z\left(\left(\frac{d}{d z} E_{\perp}\right)\left(r, \frac{z+s}{c}\right)-\nabla_{\perp} E_{z}\left(r, \frac{z+s}{c}\right)\right) .
$$

If $\boldsymbol{E}_{\perp}$ vanished at the boundaries a relation, better known as the Panofsky-WenzelTheorem [4], between the transverse and the longitudinal wake potential is obtained:

$$
\frac{\partial}{\partial s} \boldsymbol{W}_{\perp}(x, y, s)=-\nabla_{\perp} T_{\|}(x, y, s) .
$$


A integration of the transverse gradient of the longitudinal wake potential provides the transverse wake potential.

$$
W_{\perp}(x, y, s)=-\nabla_{\perp} \int_{-\infty}^{s} d s^{\prime} W_{\|}\left(x, y, s^{\prime}\right) .
$$

We now want to prove another important property of the longitudinal wake potential. $W_{\|}\left(x, y, s^{\prime}\right)$ is a harmonic function of the transverse coordinates:

$$
\nabla_{\perp}^{2} W_{\|}(x, y, s)=0 .
$$

From the Maxwell equations and the identity $\nabla \times(\nabla \times \boldsymbol{E})=\nabla(\nabla \cdot \boldsymbol{E})-\nabla^{2} \boldsymbol{E}$ we obtain an equation for $\boldsymbol{E}$.

$$
\nabla^{2} \boldsymbol{E}-\frac{1}{c^{2}} \frac{\partial^{2}}{\partial^{2} t} \boldsymbol{E}=\frac{1}{\varepsilon_{0}}\left(\nabla \rho+\frac{1}{c^{2}} \frac{\partial}{\partial t} \boldsymbol{j}\right)
$$

Since $\rho$ is a function of $z-c t$ and $j_{z}=c \rho$ the following relation holds:

$$
\frac{\partial}{\partial t} j_{z}=-c^{2} \frac{\partial}{\partial z} \rho
$$

Consequently $E_{z}$ is a solution of the wave equation, which can be written as:

$$
\nabla_{\perp}^{2} E_{z}=\left(\frac{1}{c^{2}} \frac{\partial^{2}}{\partial^{2} t}-\frac{\partial^{2}}{\partial^{2} z}\right) E_{z}
$$

For further calculation it is appropriate to use a Fourier representation of $E_{z}$ :

$$
E_{z}(x, y, z, t)=\frac{1}{(2 \pi)^{2}} \int_{-\infty}^{\infty} d \omega \int_{-\infty}^{\infty} d k_{z} e^{i\left(\omega t-k_{z} z\right)} \widetilde{E_{z}}\left(x, y, k_{z}, \omega\right) .
$$

The wave equation in Fourier space reads:

$$
\nabla_{\perp}^{2} \widetilde{E_{z}}=\left(k_{z}^{2}-(\omega / c)^{2}\right) \widetilde{E_{z}}
$$

Inserting this relation into the definition of the longitudinal wake potential yields:

$$
\begin{aligned}
\nabla_{\perp}^{2} W_{\|}(x, y, s) & =\frac{1}{q_{1}} \int_{-\infty}^{\infty} d z \nabla_{\perp}^{2} E_{z}(x, y, z,(s+z) / c) \\
& =\frac{1}{q_{1}} \int_{-\infty}^{\infty} d z \frac{1}{2 \pi^{2}} \int_{-\infty}^{\infty} d \omega \int_{-\infty}^{\infty} d k_{z} e^{i\left(\omega / c-k_{z}\right)} z\left(k_{z}^{2}-\left(\frac{\omega}{c}\right)^{2}\right) \widetilde{E}_{z}\left(x, y, k_{z}, \omega\right) \epsilon^{i(\omega / c) s} \\
& =\frac{1}{q_{1}} \frac{1}{2 \pi} \int_{-\infty}^{\infty} d \omega \int_{-\infty}^{\infty} d k_{z} e^{i\left(\omega / c-k_{z}\right) z}\left(k_{z}^{2}-\left(\frac{\omega}{c}\right)^{2}\right) \delta\left(\frac{\omega}{c}-k_{z}\right) \widetilde{E}_{z}\left(x, y, k_{z}, \omega\right) e^{i(\omega / c) s} \\
& =0
\end{aligned}
$$


We have used the Fourier representation of the $\delta$ - function.

$$
\delta\left(\frac{\omega}{c}-k_{z}\right)=\frac{1}{2 \pi} \int_{-\infty}^{\infty} d z \epsilon^{i\left(\omega / c-k_{z}\right) z} .
$$

This identity can be interpreted in our situation that only electromagnetic fields synchronous to the motion of bunch contribute to the wake potential. This is strictly only true for ultrarelativistic bunches $(v=c)$. In general we have:

$$
\nabla_{\perp}^{2} W_{\|} \sim \frac{1}{\gamma^{2}} \rightarrow 0, \text { for } \gamma \rightarrow \infty
$$

So for sufficient high energies $W_{\|}$is a harmonic function in the transverse coordinates for all positions $s$. This information will help us to evaluate the $r$ dependency of the wake potential in cylindrical symmetric structures in the next subsection.

Even for fully 3-D structures this property of $W_{\|}$leads to a numerical method to calculate the wake potential very efficiently.

\subsection{Wake Potential in Cylindrical Symmetric Structures}

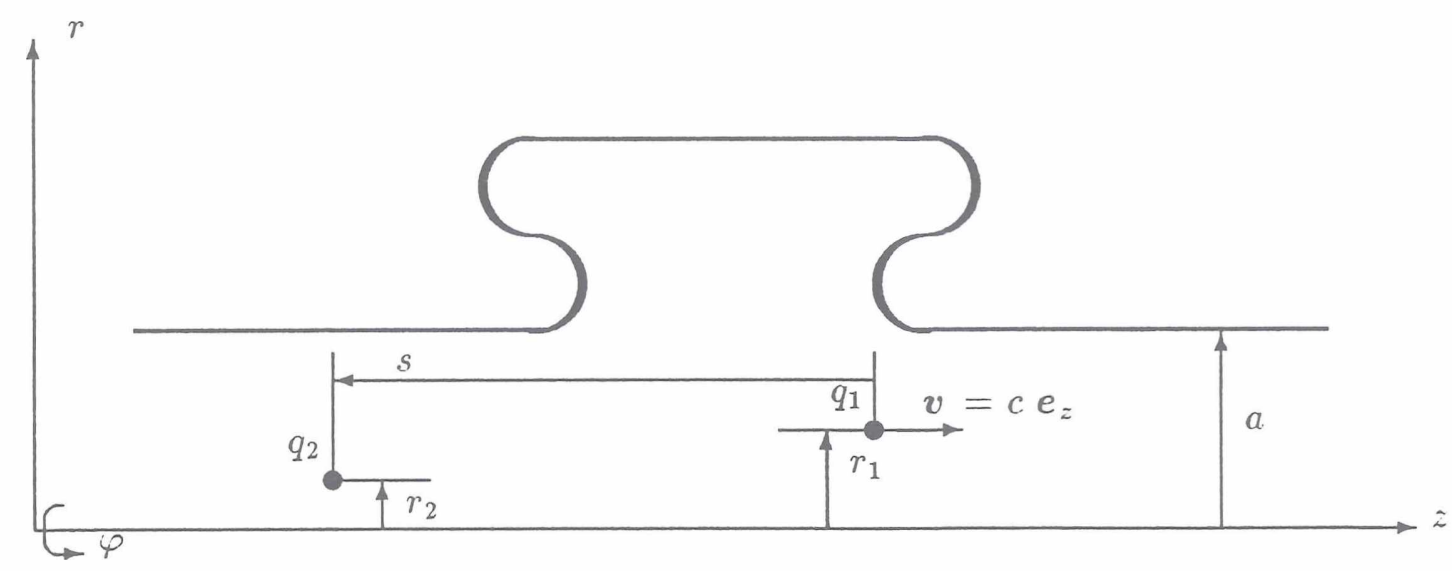

Figure 7 : A bunch with total charge $q_{1}$ traverse a cavity with offset $r_{1}$ followed by a t.est charge $q_{2}$ with offset $r_{2}$.

Consider now a cylindrical symmetric acceleration cavity with side tubes of radius $a$ (see figure 7 ). The actual shape in the region $r>a$ is of no importance for the following investigations. Two charges passed the structure from left to right at the speed of light, $q_{1}$ at a radius $r_{1}$ and $q_{2}$ a.t a radius $r_{2}$.

We want to find an expression for the net change in momentum $\Delta \boldsymbol{p}\left(r_{1}, r_{2}, \varphi_{1}, \varphi_{2}, s\right)$ experienced by $q_{2}$ due to the wake fields generated by $q_{1}$. 
Let us start with the simpler situation $r_{1}=r_{2}=r, \varphi_{1}=\varphi_{2}=\varphi$ :

$$
\Delta p_{z}(r, \varphi, s)=q_{1} q_{2} W_{\|}(r, \varphi, s)
$$

The wake potential can be expanded in a multipole series

$$
W_{\|}(r, \varphi, s)=\operatorname{Re}\left\{\sum_{m=-\infty}^{\infty} e^{i m \varphi} G_{m}(r, s)\right\}
$$

Since $W_{\|}(r, \varphi, s)$ is a harmonic function in $r, \varphi$, we have

$$
\begin{aligned}
\nabla_{\perp}^{2} W_{\|}(r, \varphi, s) & =\left(\frac{1}{r} \frac{\partial}{\partial r} r \frac{\partial}{\partial r}+\frac{1}{r^{2}} \frac{\partial^{2}}{\partial \varphi^{2}}\right) W_{\|}(r, \varphi, s) \\
& =\operatorname{Re}\left\{\sum_{m=-\infty}^{\infty} e^{i m \varphi}\left(\frac{1}{r} \frac{\partial}{\partial r} r \frac{\partial}{\partial r}-\frac{m^{2}}{r^{2}}\right) G_{m}(r, s)\right\} \\
& =0
\end{aligned}
$$

So for all $m$ the expansion functions $G_{m}(r, s)$ have to fulfill the Poisson equation:

$$
\frac{1}{r} \frac{\partial}{\partial r}\left(r \frac{\partial}{\partial r} G_{m}(r, s)\right)-\frac{m^{2}}{r^{2}} G_{m}(r, s)=0
$$

The solutions are:

$$
\begin{aligned}
G_{0}(r, s) & =U_{0}(s)+V_{0}(s) \ln r \\
G_{m}(r, s) & =U_{m}(s) r^{m}+V_{m}(s) r^{-m} \quad \text { for } m>0 .
\end{aligned}
$$

Keeping only the at the origin $(r=0)$ regular solutions the longitudinal wake potential can be written as

$$
W_{\|}(r, \varphi, s)=\sum_{m=0}^{\infty} r^{m} u_{m}(s) \cos m \varphi,
$$

with expansion functions $u_{m}(s)$ depending on the details of the given cavity geometry.

The above relation describes the momentum change $\Delta p_{z}=q_{1} q_{2} W_{\|}$due to fields that are present before the charge $q_{2}$ enters. From conversation of energy it follows that the fields generated by the charge $q_{1}$ get the energy out of the longitudinal momentum, since $r_{1}$ and $r_{2}$ were defined to be constant. Thus it follows for the general case of a charge $q_{1}$ at $r=r_{1}$ generating the fields that act on a second charge $q_{2}$ at $r=r_{2}$ that the longitudinal wake potential is given by:

$$
W_{\|}\left(r_{1}, r_{2}, \varphi_{1}, \varphi_{2}, s\right)=\sum_{m=0}^{\infty} r_{1}^{m} r_{2}^{m} w_{m}(s) \cos m\left(\varphi_{2}-\varphi_{1}\right) .
$$


The transverse wake potential is according to the Panofsky Wenzel Theorem given by:

$$
\begin{aligned}
W_{\perp}\left(r_{1}, r_{2}, \varphi_{1}, \varphi_{2}, s\right)= & -\left(e_{r} \frac{\partial}{\partial r_{2}}+e_{\varphi} \frac{1}{r_{2}} \frac{\partial}{\partial \varphi_{2}}\right) \int_{-\infty}^{s} d s^{\prime} W_{\|}\left(r_{1}, r_{2}, \varphi_{1}, \varphi_{2}, s^{\prime}\right) \\
= & \sum_{m=0}^{\infty}\left\{-e_{r} m r_{1}^{m} r_{2}^{m-1} \int_{-\infty}^{s} d s^{\prime} w_{m}\left(s^{\prime}\right) \cos m\left(\varphi_{2}-\varphi_{1}\right)\right. \\
& \left.\quad+e_{\varphi} m r_{1}{ }^{m} r_{2}{ }^{m-1} \cdot \int_{-\infty}^{s} d s^{\prime} w_{m}\left(s^{\prime}\right) \sin m\left(\varphi_{2}-\varphi_{1}\right)\right\}
\end{aligned}
$$

The characteristic wake functions $w_{m}(s)$ have to be calculated by solving Maxwell's equations for the given geometry and initial conditions:

$$
w_{m}(s)=\frac{\left.\int_{-\infty}^{\infty} d z E_{z m}\left(r_{2}, \varphi_{2}, z,(z+s) / c\right)\right)}{r_{1}^{m} r_{2}^{m} \cos m\left(\varphi_{2}-\varphi_{1}\right)}
$$

Normally a computer code like TBCI is used to do this job.

Since the dependency on the radial position is now known the integration can be performed for any radial position, especially for the tube radius $r=a$. Since $E_{z}$ vanished at the metallic tube boundary only the cavity gap contributes to the integral. The explicit knowledge of the wake potential for different radial positions enables to transform a originally infinite integration range to a finite range over the cavity gap.

It should be mentioned that for many practical cases, due to the $(r / a)^{m}$ dependency, the longitudinal wake potential is dominated by the monopole term, while the transverse is by the dipole term:

$$
\begin{aligned}
W_{\|}\left(r_{1}, r_{2}, \varphi_{1}, \varphi_{2}, s\right) & =w_{0}(s) \cos m\left(\varphi_{2}-\varphi_{1}\right) \\
W_{\perp}\left(r_{1}, r_{2}, \varphi_{1}, \varphi_{2}, s\right) & =r_{1} \int_{-\infty}^{s} d s^{\prime} w_{1}\left(s^{\prime}\right) \quad\left(-e_{r} \cos m\left(\varphi_{2}-\varphi_{1}\right)+e_{\varphi} \sin m\left(\varphi_{2}-\varphi_{1}\right)\right) .
\end{aligned}
$$

\subsection{Fully 3-D Structures}

While for cylindrical symmetric structures the dependency of the wake potential on the transverse coordinate is known, in the general situation only numerical methods can yield the results. since even for the general situation $W_{\|}$is a harmonic function

$$
\nabla_{\perp}^{2} W_{\|}(x, y, s)=0
$$

it is possible to find $W_{\|}(x, y, s)$ for all possible beam positions $(x, y)$ by a numerical solution of Poissons equation if one knows the wake potential on the boundary. This indirect method has the advantage that the integration domain for each test beam on the boundary is finite (see figure 2.5). 

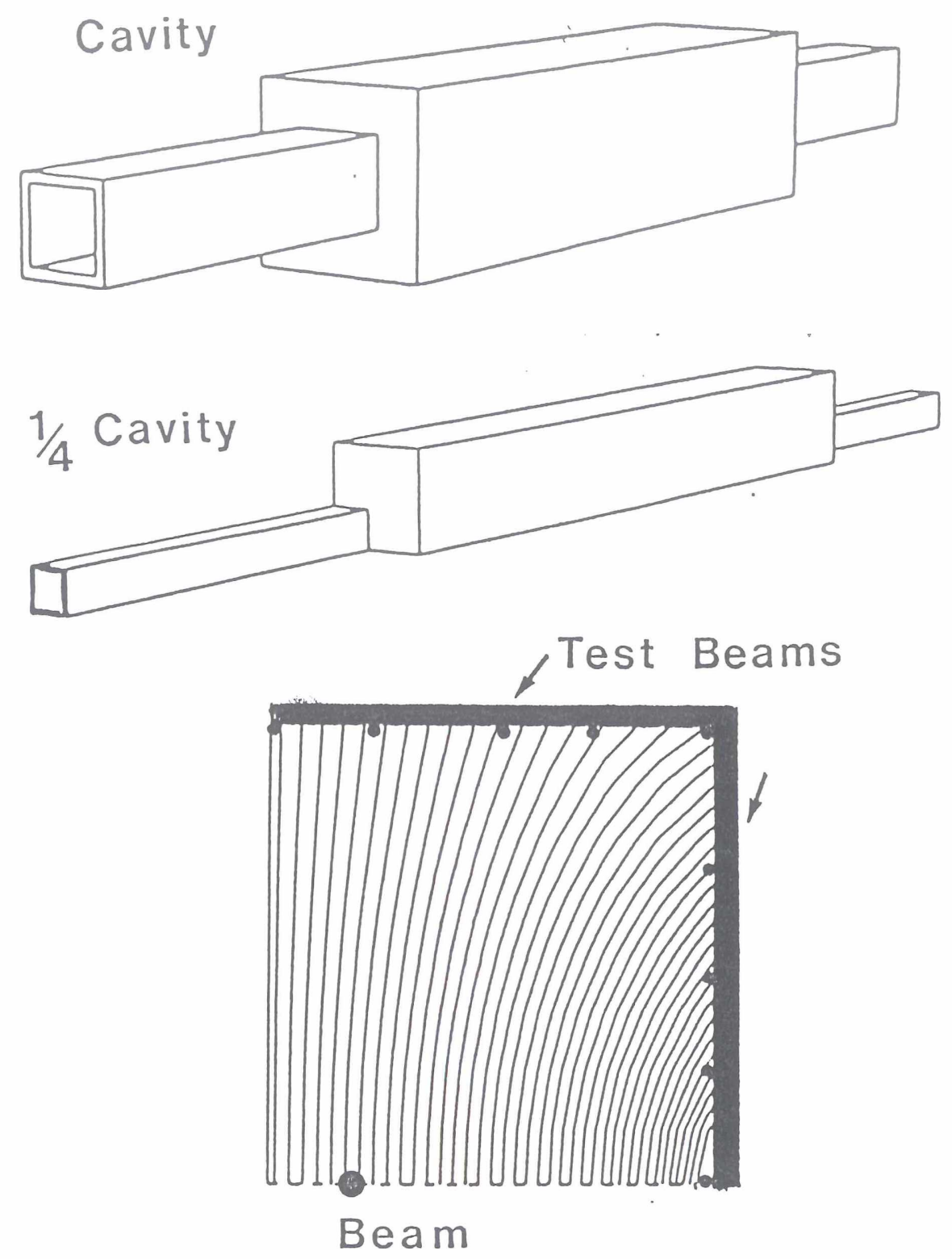

Figure 8: 3-D Cavity structure with 2 symmetry planes. Due to the symmetries it is sufficient to treat only $1 / 4$ cavity numerically. The third picture shows lines of constant longitudinal wake potential. 
The transverse wake potential can be calculated from the longitudinal one using the Panofsky Wenzel Theorem. Figure 9 shows the transverse gradient of the longitudinal wake potential in a beam tube.

The boundary values (test beams) on the tube radius are found by a numerical integration of the Maxwell equations. See the section about analytical and numerical calculations of wake fields for more details.

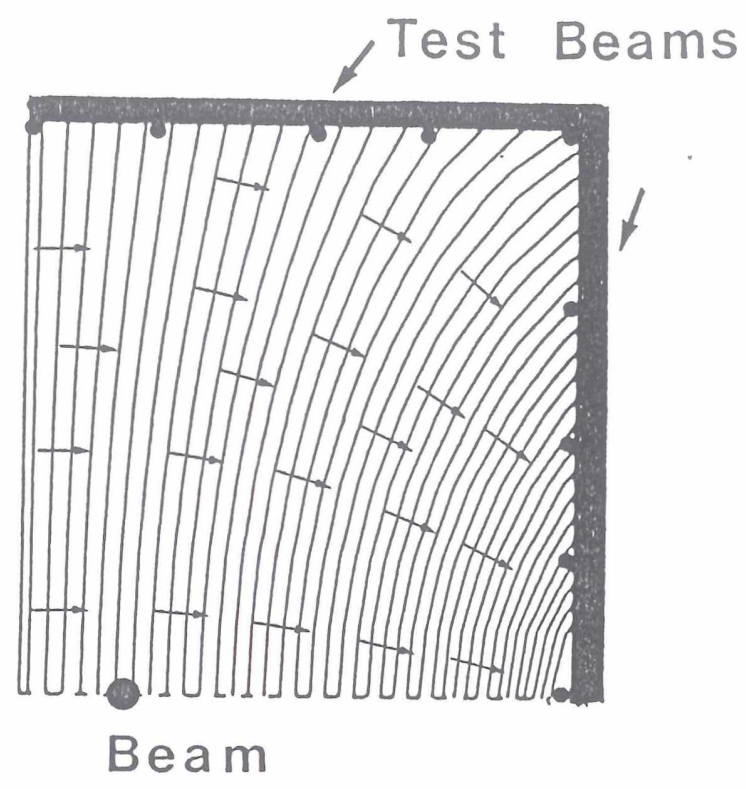

Figure 9: The picture show the gradient of the longitudinal wake potential. An integration provides the transverse wake potential according to Panofsky Wenzel Theorem. 


\section{Impedances}

\subsection{Definitions}

The Fourier transform of the wake potential is called the impedance or coupling impedance.

$$
Z_{\|}(x, y, \omega)=\frac{1}{c} \int_{-\infty}^{\infty} d s W_{\|}(x, y, s) \exp \left(-i \frac{\omega}{c} s\right)
$$

Wake potential and impedance are two descriptions of the same thing, the coupling between the beam and its environment. The wake potential is the time domain description, the impedance the frequency domain description. The reason for the usefulness of the impedance is that it often contains a number of sharply defined frequencies corresponding to the modes of the cavity or the long range part of the wake potential. Figure 3.1 shows the real part of a typical impedance for a cavity. Below the cut off frequency of the beam pipe there are for each cavity mode a sharp peak. Above the cut off frequency a continuous spectrum of beam pipe modes contribute to the impedance. These are important for the short range wake field effects over the bunch, especial for very short bunches.

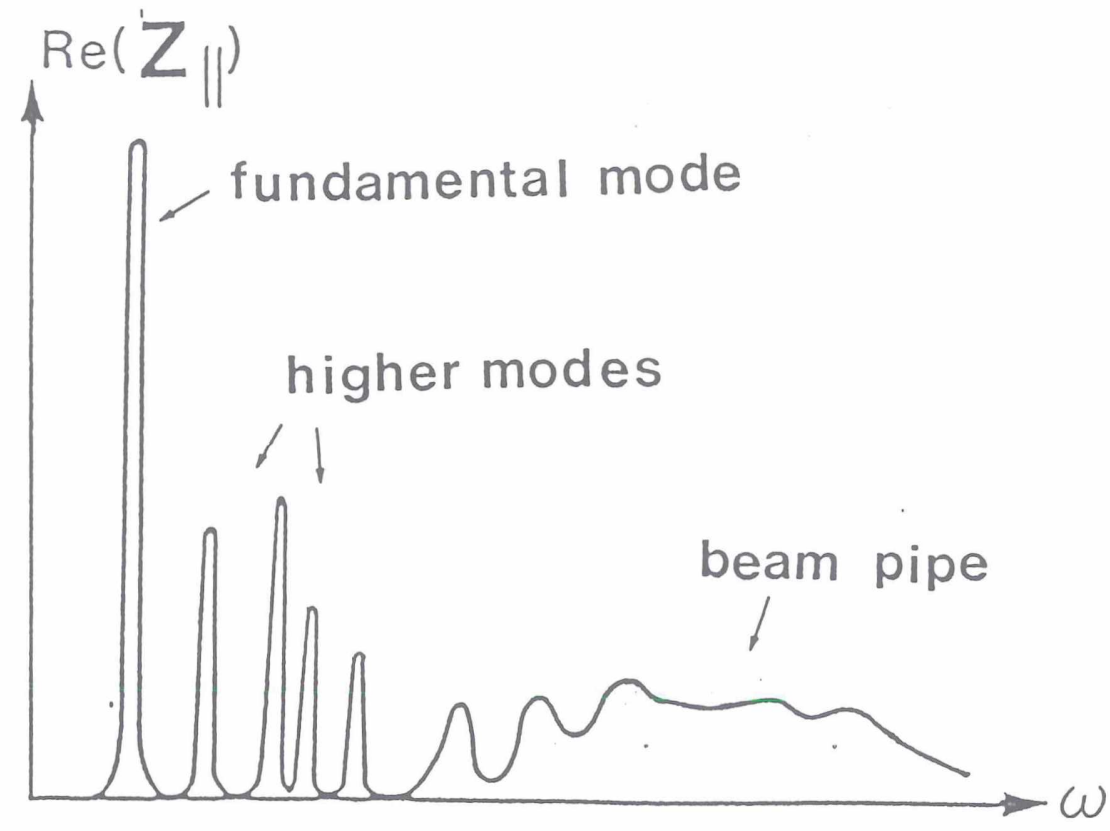

Figure 10: Real part of the impedance for a cavity with side pipes. The sharp peaks correspond to cavity modes while above the cut off frequency the continuous spectrum of beam pipe modes contribute to the impedance. 
For the transverse impedance it is often convenient to use a definition with an extra factor $-i$ :

$$
Z_{\perp}(x, y, \omega)=\frac{-i}{c} \int_{-\infty}^{\infty} d s W_{\perp}(x, y, s) \exp \left(-i \frac{\omega}{c} s\right) .
$$

The reason is that then the Panofsky Wenzel Theorem reads in the frequency domain:

$$
\frac{\omega}{c} Z_{\perp}(x, y, \omega)=\nabla_{\perp} Z_{\|}(x, y, \omega)
$$

We should mention some properties of the impedance here. Firstly, since the wake potential is a real function the real part of the impedance is an even function of the frequency while the imaginary part is an odd function of it.

$$
\operatorname{Re}\left\{Z_{\|}(\omega)\right\}=\operatorname{Re}\left\{Z_{\|}(-\omega)\right\} \quad \operatorname{Im}\left\{Z_{\|}(\omega)\right\}=-\operatorname{Im}\left\{Z_{\|}(-\omega)\right\} .
$$

Hence we have for the wake potential in terms of the impedance:

$$
\begin{aligned}
W_{\|}(s) & =\frac{1}{2 \pi} \int_{-\infty}^{\infty} d \omega Z_{\|}(\omega) \exp \left(i \frac{\omega}{c} s\right) \\
& =\frac{1}{2 \pi} \int_{-\infty}^{\infty} d \omega\left(\operatorname{Re}\left\{Z_{\|}(\omega)\right\} \cos \left(\frac{\omega}{c} s\right)-\operatorname{Im}\left\{Z_{\|}(\omega)\right\} \sin \left(\frac{\omega}{c} s\right)\right)
\end{aligned}
$$

Furthermore due to causality $\operatorname{Re}\left\{Z_{\|}(\omega)\right\}$ and $\operatorname{Im}\left\{Z_{\|}(\omega)\right\}$ are not independent of each other. From $W_{\|}(-s)=0$ for all $s>0$ it follows:

$$
\int_{-\infty}^{\infty} d \omega \operatorname{Re}\left\{Z_{\|}(\omega)\right\} \cos \left(\frac{\omega}{c} s\right)=-\int_{-\infty}^{\infty} d \omega \operatorname{Im}\left\{Z_{\|}(\omega)\right\} \sin \left(\frac{\omega}{c} s\right) \quad \text { for all } s>0 .
$$

So only the real (or imaginary) part of the impedance is really needed.

$$
W_{\|}(s)=\frac{1}{\pi} \int_{-\infty}^{\infty} d \omega \operatorname{Re}\left\{Z_{\|}(\omega)\right\} \cos \left(\frac{\omega}{c} s\right) .
$$

In the next section the energy losses of the bean to the different modes of a cavity are studied in detail. This will lead to a method to calculated some parts of the impedance.

\subsection{Loss Parameters}

Consider a closed pill box cavity (see figure 11). The electric field is an infinite series of eigen modes of the cavity ringing with the frequencies $\omega_{n}=2 \pi f_{n}$.

$$
\boldsymbol{E}(\boldsymbol{r}, t)=\operatorname{Re}\left\{\sum_{n=0}^{\infty} \boldsymbol{E}_{n}(\boldsymbol{r}) \epsilon^{i \omega_{n} t}\right\}
$$

We want to study the coupling between the bean and the various modes. A point. charge traversing the cavity with the speed of light $(z=c t)$ " sees "the (complex) voltage:

$$
T_{n}^{\gamma}=\int_{-\infty}^{\infty} d z E_{z, n}(z) c^{i \omega_{n} z / c}
$$




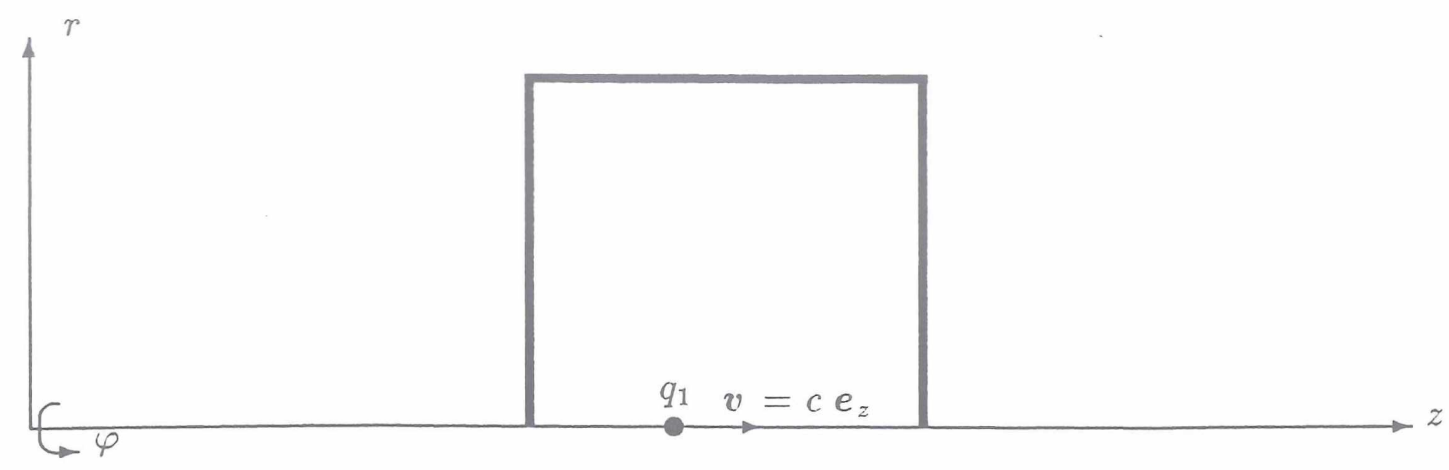

Figure 11: Closed pill box cavity excited by a point charge $q_{1}$

The total field energy stored in mode $n$ is given by

$$
U_{n}=\frac{\epsilon_{0}}{2} \int d^{3} r \mid \boldsymbol{E}_{n}\left(\left.\boldsymbol{r} \cdot\right|^{2}\right.
$$

We now define the loss parameter $k_{n}$.

$$
k_{n}=\frac{\left|V_{n}\right|^{2}}{4 U_{n}}
$$

is a phase and amplitude independent quantity.

The wake potential for $s>0$ of a point charge is given by (see [5] or Appendix A)

$$
W_{\|}^{\delta}(s)=\sum_{n=0}^{\infty} 2 k_{n} \cos \left(\omega_{n} s / c\right) \text {. }
$$

In the next section we will show that the self wake is only one half of the wake directly behind the point charge (fundamental theorem of beam loading). So the energy lost of the point charge into the mode $n$ is given by:

$$
\Delta W_{n}=q_{1}^{2} k_{n}
$$

The wake potential of a Gaussian bunch with charge density:

$$
\rho(\boldsymbol{r}, t)=q_{1} \lambda(z-c t), \quad \lambda(s)=\frac{1}{\sigma \sqrt{2 \pi}} \exp \left(-\frac{\left(s-s_{0}\right)^{2}}{2 \sigma^{2}}\right)
$$

is obtained by a convolution integral with the point charge wake potential $W_{\|}^{\delta}$.

$$
W_{\|}(s)=\int_{0}^{\infty} d s^{\prime} \lambda\left(s-s^{\prime}\right) W_{\|}^{\delta}\left(s^{\prime}\right)
$$


For $\sigma \ll s-s_{0}$ the result of convolution integral is

$$
W_{\|}^{\delta}(s)=\sum_{n=0}^{\infty} 2 k_{n} \exp \left(-\frac{1}{2}\left(\frac{\omega_{n}}{c}\right)^{2} \sigma^{2}\right) \cos \left(\omega_{n} s / c\right) .
$$

It is now possible to define various loss parameters for the coupling of the beam with the different modes of the cavity.

Total loss parameter

$$
k_{\text {tot }}=\int_{-\infty}^{\infty} d s \lambda(s) W_{\|}(s)
$$

$\Delta W=q_{1}{ }^{2} k_{\text {tot }}$ is the total energy lost of the Gaussian bunch. All modes of the cavity and all wave guide modes are included.

Loss to the fundamental mode

$$
k_{f u}=k_{0} \exp \left(-\frac{1}{2}\left(\frac{\omega_{n}}{c}\right)^{2} \sigma^{2}\right)
$$

This parameter describes the desired coupling between the fundamental cavity mode and the beam.

Loss to higher order modes

$$
k_{h m}=\sum_{n=1}^{N} 2 k_{n} \exp \left(-\frac{1}{2}\left(\frac{\omega_{n}}{c}\right)^{2} \sigma^{2}\right)
$$

Beside the fundamental mode there are some $(N)$ resonant cavity modes.

\section{Loss into the beam pipe}

$$
k_{\text {tube }}=k_{\text {tot }}-k_{f u}-k_{h m}
$$

The beam can also lost energy into wave guide modes of the side tubes. Especially very short bunches will excite that kind of modes in the continuous spectrum of the structure.

\section{Total parasitic loss}

$$
\begin{aligned}
k_{p a r} & =k_{t o t}-k_{f u} \\
& =k_{t u b \epsilon}+k_{h m}
\end{aligned}
$$

All parasitic effects are summarized in the loss parameter $k_{\text {par }}$.

Figure 3.2 shows all the above defined loss parameters for a single cell of a PETRA cavity as a function of the bunch length $\sigma$. For very short bunches parasitic losses are dominant. Consequently a careful cavity design requires always a closer look at higher order mode effects and tube radiation losses. For a further discussion see the section about strategies in accelerator design. 


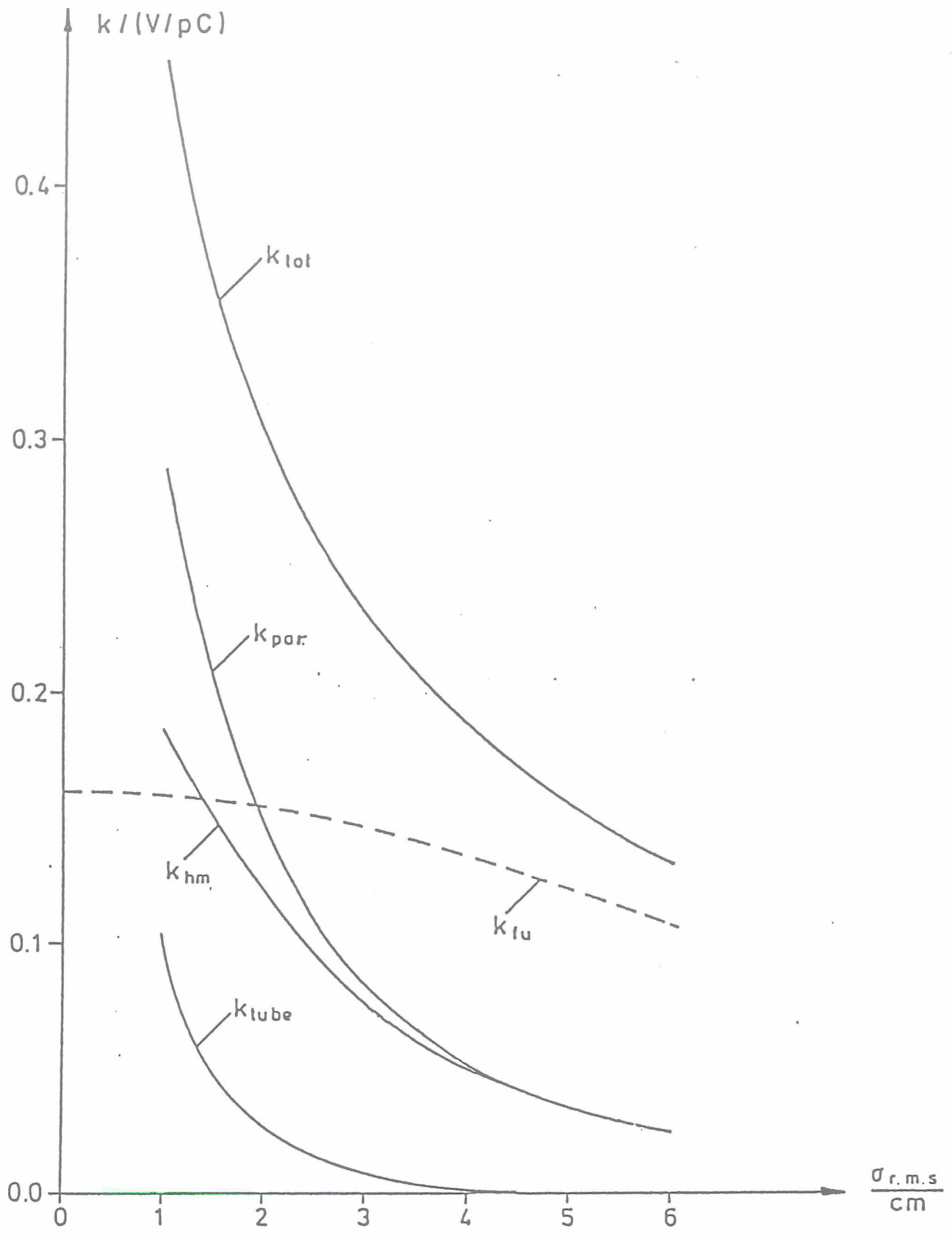

The loss parameter $k_{101}, k_{\text {por }} k_{h m}, k_{\text {lube }}$ and $k_{\text {lu }}$ as a function of $\sigma_{r . m . s}$ (Gaussian bunch) for a single cell of a PETRA cavily

Figure 12: Loss parameters 


\subsection{Fundamental Theorem of Beam Loading}

We already mentioned that the self wake is only one half of the wake directly behind the point charge $q_{1}$. In this section the coupling of a point charge to one cavity mode ringing with frequency $\omega=1 \pi f$ is studied in detail.

The induced voltage behind the point charge $q_{1}$ is

$$
V(s)=\hat{V} \cos (\omega s / c) \quad s>0,
$$

with $\hat{V} \sim q$. The dependency on $s$ is a pure cos - function without any extra phase. Otherwise a second point charge $q$ following in an appropriate distance could gain more energy out of the cavity mode than the first point charge lost into that mode. That would lead clearly to a contradiction to the conversation of energy.

Now consider two point charges with the same charge $q$ following each other in a distant $\lambda / 2\left(\lambda=c / f\right.$ the wavelength of the mode). Let $V_{1}$ and $V_{2}$ be the induced voltages by charge \# 1 and \# 2 (see figure 3.3 ).

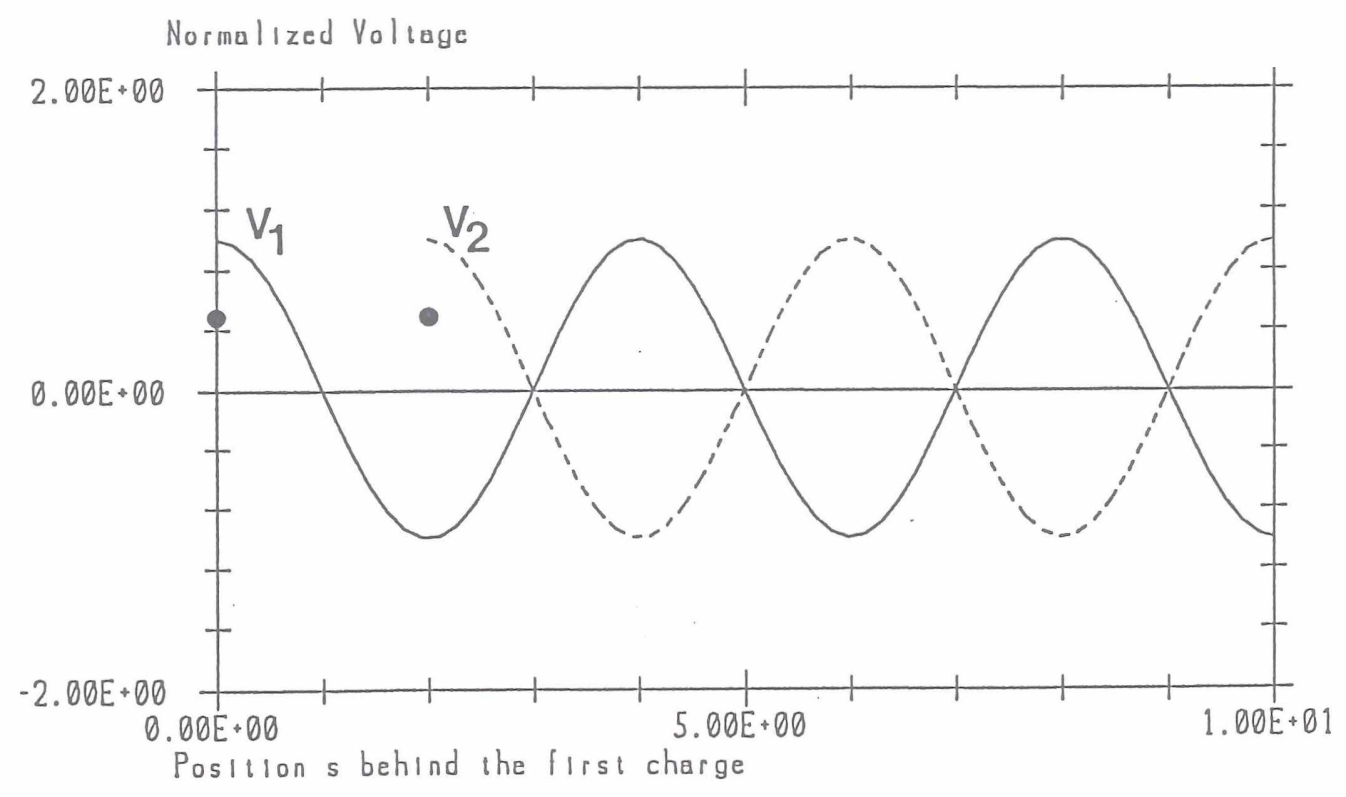

Figure 13: Induced voltages by point charge \# 1 and \# 2.

The point charge \# 1 lost the energy $\Delta W_{1}$ into the cavity mode:

$$
\Delta T_{1}^{\top}=k q^{2}=q \eta V_{1}
$$

$\eta$ is an unknown factor describing the difference between the self wake and the wake directly behind the charge. 
Since the second charge followed in a distant $\lambda / 2$ it takes the whole stored energy out of the mode:

$$
\begin{aligned}
\Delta W_{2} & =\Delta W_{1}=k q^{2} \\
& =q\left(V_{1}-\eta V_{2}\right)
\end{aligned}
$$

This provides the following equation for the unknown factor $\eta$.

$$
q \eta V_{1}=q\left(V_{1}-\eta V_{2}\right)
$$

Since $V_{1}=V_{2}$ for identical point charges we obtain

$$
\eta=\frac{1}{2}
$$

Consequently the induced voltage by a point charge is given by:

$$
V(s)= \begin{cases}2 q k \cos (\omega s / c) & s>0 \\ q k & s=0 \\ 0 & s<0\end{cases}
$$

The energy lost into the cavity mode is

$$
\Delta W=q V(0)=q^{2} h
$$

\subsection{Shunt Impedance and Quality Factor}

Various loss parameters were defined to describe the energy lost of the beam to cavity modes. But once excited by a beam such a mode is not ringing forever. Due to the finite conductivity of the cavity walls the fields amplitudes are slowly reduced. The energy in the cavity modes is transformed into heat.

The wall currents are induced in a thin surface layer characterized by the skin depth.

$$
\delta=\sqrt{\frac{2}{\omega \mu \kappa}}
$$

$\omega=2 \pi f$ is the frequency of the mode, $\kappa$ the conductivity of the wall material and $\mu$. the permeability.

The wall losses can be calculated approximately from the fields obtained for the modes established for wall with infinite conductivity. If $\boldsymbol{H}_{t}$ is the magnetic field tangential to the surface the total power dissipated into the walls is given by a surface integral

$$
P=\frac{1}{2} \int_{\partial V} d^{2} r \frac{\left|\boldsymbol{H}_{t}\right|^{2}}{\kappa \delta},
$$


While the energy stored in the mode is

$$
U=\frac{\epsilon_{0}}{2} \int_{V} d^{3} r|\boldsymbol{E}|^{2}
$$

The quality factor of a cavity mode is defined as

$$
Q=\frac{\omega U}{P}=\frac{2 \pi}{T} \frac{\text { stored energy }}{\text { powerloss }}
$$

Typical values for copper cavities are:

$$
\begin{array}{ll}
Q \approx 10000 & f=3 \mathrm{GHz} \\
Q \approx 35000 & f=500 \mathrm{MHz}
\end{array}
$$

The connection with the attenuation constant $\alpha$ for the fields are

$$
\alpha=\frac{\omega}{2 Q}, \quad \boldsymbol{H}, \boldsymbol{E} \sim e^{-\alpha t}
$$

Since $U \sim E^{2} \sim e^{-\alpha t}$ we have

$$
P=\frac{d}{d t} U=-2 \alpha \cdot U
$$

The induced voltage by a point charge $q$ into a mode with frequency $\omega$ has to be modified for long distances $s$

$$
V(s)=2 q k \cos (\omega s / c) \exp \left(-\frac{\omega}{2 Q} \frac{s}{c}\right)
$$

Although the induced voltage $V(s)$ is completely determined by the parameters $k, Q$ and $\omega$ another quantity the shunt impedance $R$ is commonly used. Formally it can be defined by

$$
R=\frac{4 k Q}{\omega} \quad(\mathrm{US}) \quad \text { or } \quad R=\frac{2 k Q}{\omega} \quad \text { (electrical eng.) }
$$

Their meaning will become obvious in the following example. Consider a circular accelerator with one cavity and (say) four identical bunches following each other in a distant $T_{1}$ in time (see figure 3.4 ).

The induced voltage in one cavity mode after $n$ bunches have traversed the cavity is:

$$
\hat{V}(j)=\sum_{j=1}^{n} 2 k q \cos \left(\omega j T_{1}\right) \exp \left(-\frac{j T_{1} \omega}{2 Q}\right)
$$

Let us now assume the worst case that all bunches are in phase with the cavity mode

$$
\omega T_{1}=2 \pi
$$




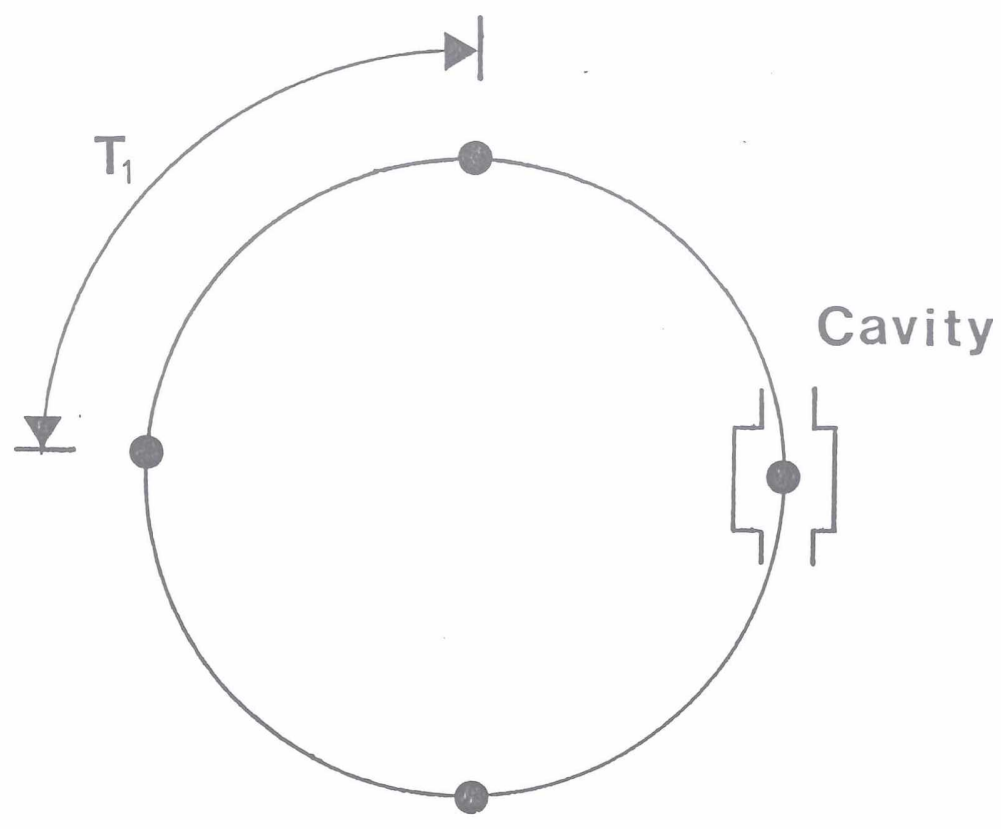

Figure 14: Circular accelerator with one cavity successively transversed by four bunches.

In the limit $n \rightarrow \infty$ we obtain

$$
\begin{aligned}
\hat{V}(\infty) & =2 k q \sum_{j=1}^{\infty} \exp \left(\frac{\omega j T_{1}}{2 Q}\right. \\
& =2 k q \frac{1}{1-\exp \left(-\omega T_{1} /(2 Q)\right)} \\
& \approx 2 k q \frac{2 Q}{\omega T_{1}} .
\end{aligned}
$$

The long range wake amplitude of one mode including losses due to wall currents is

$$
\begin{aligned}
\hat{V}(\infty) & =\frac{4 k Q}{\omega} \frac{q}{T_{1}} \\
& =R I .
\end{aligned}
$$

This result is of the form voltage equal shunt impedance times current.

Typical values for a $500 \mathrm{MHz}$ copper cavity are:

$$
R=20 \frac{\mathrm{M} \Omega}{\mathrm{m}}
$$

( US shunt impedance definition). 


\section{Analytical and Numerical Calculations of Wake Fields}

\subsection{Analytical Calculation for a Pill Box}

For a closed pill box cavity all modes can be calculated analytically [14]. Consider a pill box with radius $R$ and gap length $g$ (see figure 15). The normalized electromagnetic

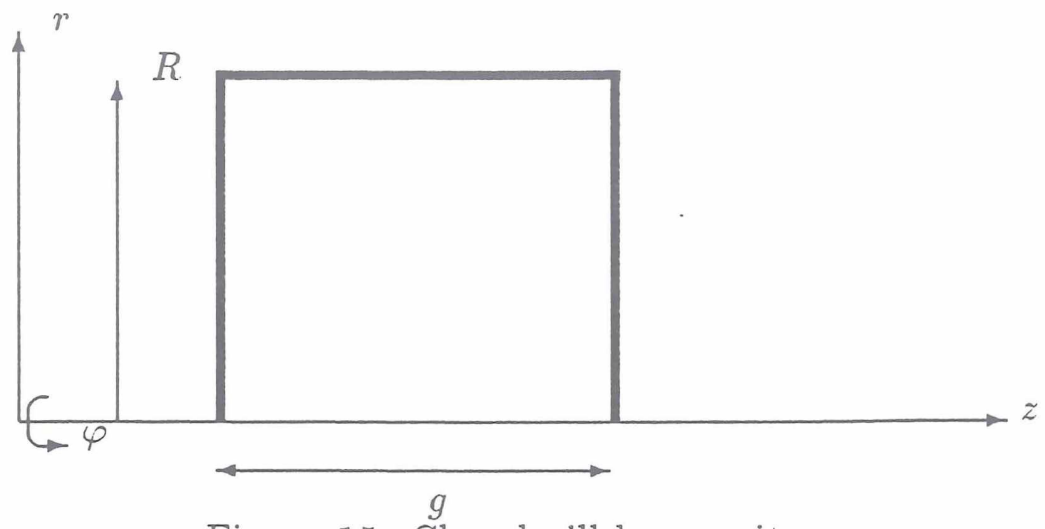

Figure 15: Closed pill box cavity

field of the monopole modes numbered by $n, p$ is given by:

$$
\begin{aligned}
E_{z}^{(n, p)} & =\frac{j_{n}}{R} J_{0}\left(j_{n} \frac{r}{R}\right) \cos \left(\frac{\pi p z}{g}\right) \exp \left(i \omega_{n, p} t\right) \\
E_{r}^{(n, p)} & =\frac{\pi p}{g} J_{1}\left(j_{n} \frac{r}{R}\right) \sin \left(\frac{\pi p z}{g}\right) \exp \left(i \omega_{n, p} t\right) \\
H_{\phi}^{(n, p)} & =i \omega_{n, p} \epsilon_{0} J_{1}\left(j_{n} \frac{r}{R}\right) \cos \left(\frac{\pi p z}{g}\right) \exp \left(i \omega_{n, p} t\right),
\end{aligned}
$$

where $j_{n}$ is the $\mathrm{n}^{\text {th }}$ zero of the Bessel function $J_{0}(x)$ and

$$
\left(\frac{\omega_{n, p}}{c}\right)^{2}=\left(\frac{j_{n}}{R}\right)^{2}+\left(\frac{\pi p}{g}\right)^{2} .
$$

Hence the voltage becomes

$$
\begin{aligned}
V_{n, p} & =\int_{z}^{g} d z E_{z}(r=0, z, t=z / c) \\
& =\frac{i \omega_{n, p} R}{j_{n} c}\left(1-(-1)^{p} \exp \left(i \omega_{n, p} g / c\right)\right)
\end{aligned}
$$

The stored energy is given by:

$$
U_{n, p}=\frac{\mu_{0}}{2} \int_{0}^{R} d r r \int_{0}^{2 \pi} d \varphi \int_{0}^{g} d \approx H_{\varphi}^{(n, p)}\left(H_{\varphi}^{(n, p)}\right)^{*}
$$




$$
=\frac{\pi \epsilon_{0}}{4}\left(\frac{\omega_{n, p}}{c}\right)^{2} g R^{2} J_{1}^{2}\left(j_{n}\right)
$$

The loss parameters can now be calculated

$$
\begin{aligned}
k_{n, p} & =\frac{V_{n, p} V_{n, p}^{*}}{4 U_{n, p}} \\
& =\frac{1}{\pi \epsilon_{0} g} \frac{2}{1+\delta_{0, p}} \frac{1-(-1)^{p} \cos \left(\omega_{n, p} g / c\right)}{j_{n}^{2} J_{1}^{2}\left(j_{n}\right)}
\end{aligned}
$$

The expression for the point charge $(q)$ monopole wake potential becomes:

$$
W_{\|}^{\delta}(s)=2 q \sum_{n=1}^{\infty} \sum_{p=0}^{\infty} k_{n, p} \cos \left(\omega_{n, p} s / c\right) .
$$

The wake potential is the sum of all voltages induced in all modes.

The wake potential of a Gaussian bunch with charge density:

$$
\rho(\boldsymbol{r}, t)=q_{1} \lambda(z-c t), \quad \lambda(s)=\frac{1}{\sigma \sqrt{2 \pi}} \exp \left(-\frac{\left(s-s_{0}\right)^{2}}{2 \sigma^{2}}\right)
$$

is given by

$$
W_{\|}(s)=\int_{0}^{\infty} d s^{\prime} \lambda\left(s-s^{\prime}\right) W_{\|}^{\delta}\left(s^{\prime}\right)
$$

Figure 16 shows the wake potential of a Gaussian bunch $(\sigma=2.5 \mathrm{~cm})$ due to a pill box cavity $(R=5 \mathrm{~cm}, g=10 \mathrm{~cm})$. The dashed line is obtained from a mode analysis result based on 40 modes, while the solid line is calculated with the computer code TBCI with a $11 \times 21$ mesh.

Figure 16 shows a good agreement between the two different methods for the long range $(s \gg \sigma)$ wake potential. But even for a simple example as a pill box cavity it is very hard to compute the wake potential by a mode analysis since many modes are needed. The reason is that for $s$ inside the bunch the charge distribution contributing to the wake potential is cut off due to causality. Hence the Fourier spectrum of the charge distribution contains many (say > 1000) modes. Since an accelerator is not made of closed boxes the mode analysis is not sufficient to calculate the wake potential. The continuous spectrum of wave guide modes in the beam pipes contribute also to the impedance, especially to the short range wake.

Figure 17 shows the real part of the impedance for a PETRA cavity. Above the cut off frequency $f_{c}$ the spectrum is continuous. 


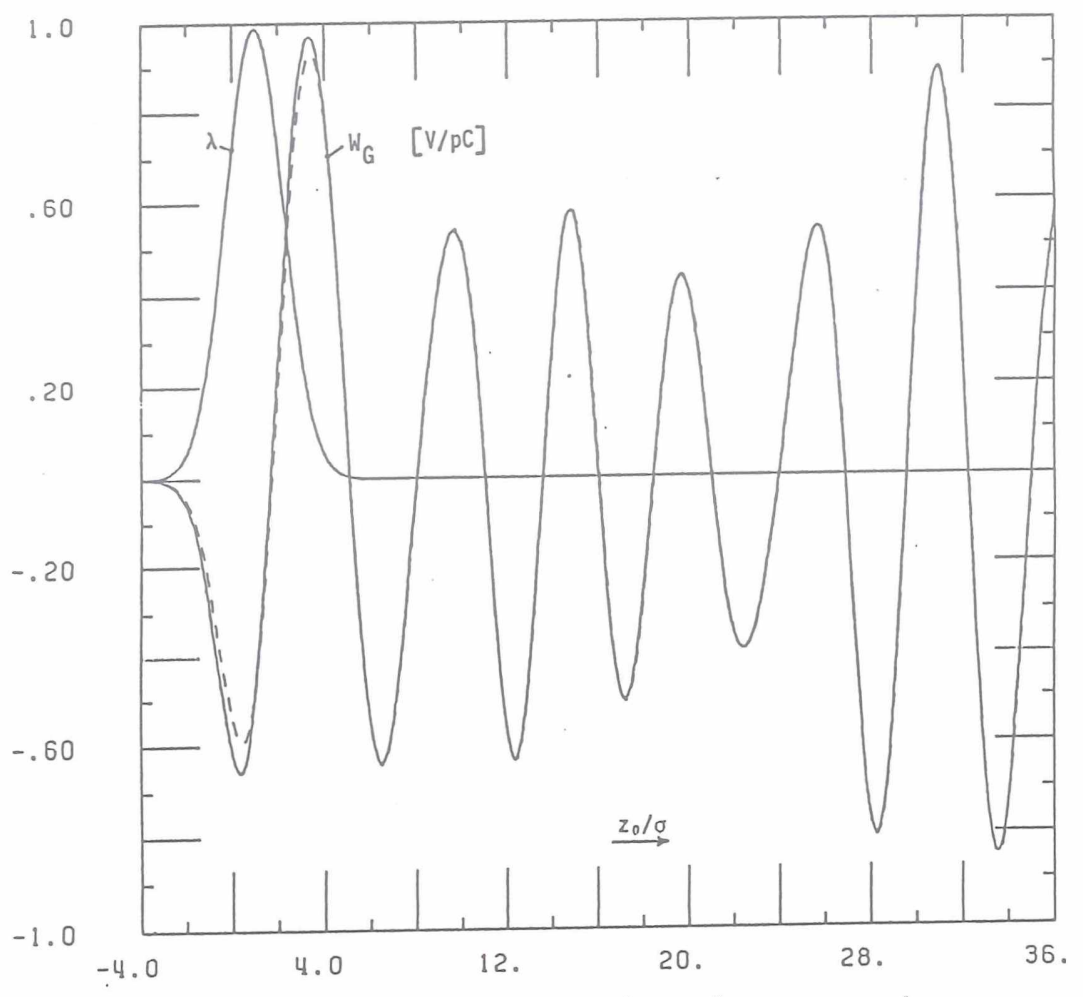

Figure 16: Normalized wake potential of a Gaussian bunch due to a pill box cavity for $-4 \sigma \leq s \leq 36 \sigma$. The dashed line shows a mode analysis result (40 modes), while the solid line is obtained with TBCI on a $11 \times 21$ mesh.

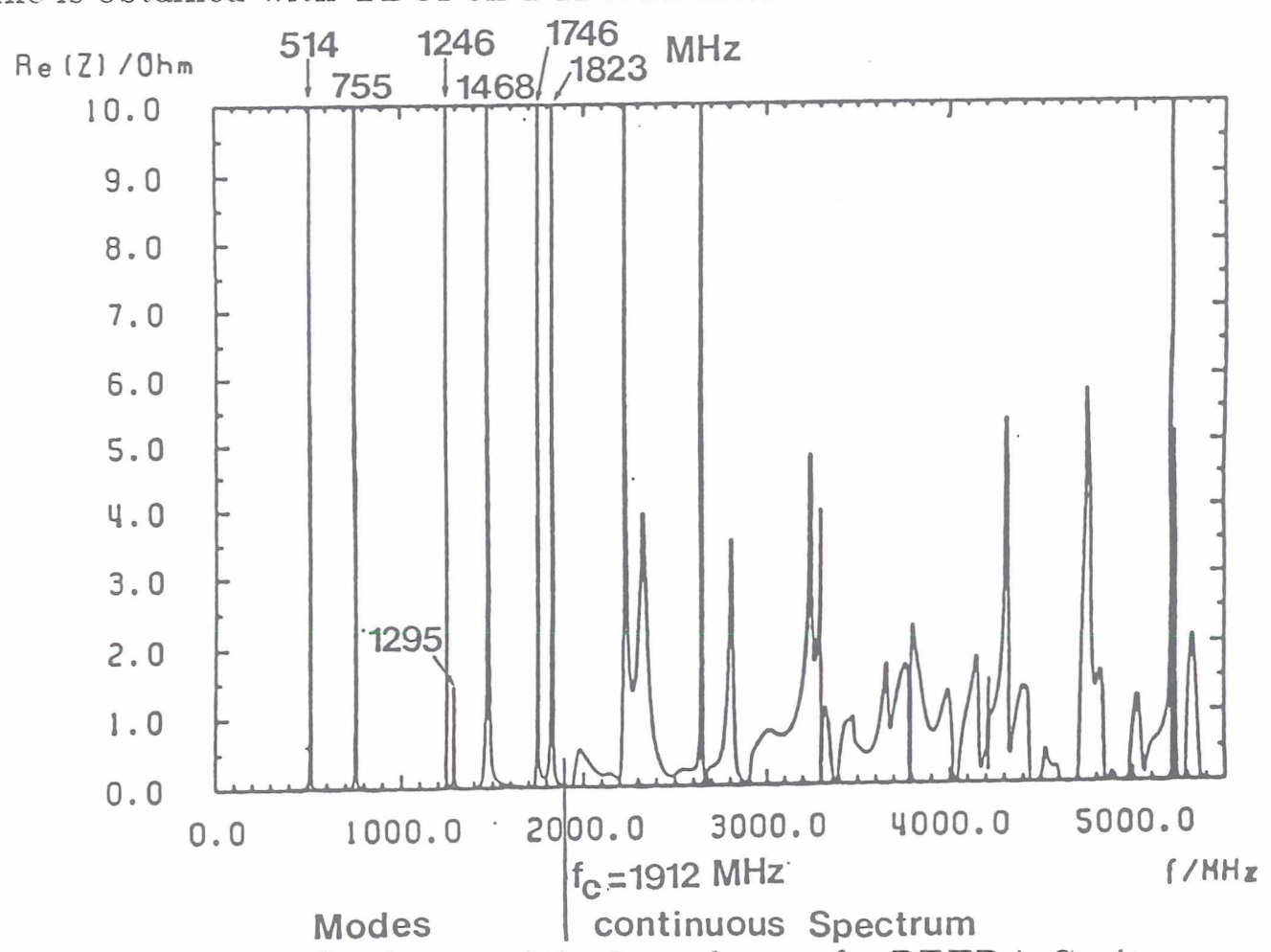

Figure 17: Real part of the Impedance of a PETRA Cavity 


\subsection{Numerical Calculations}

\subsubsection{Grid Maxwell Equations}

A numerical approach for the solution of Maxwell's equations is presented. Based on a finite difference lattice the method transforms each of the four Maxwell equations into an equivalent matrix expression that can be subsequently treated by matrix mathematics and suitable numerical methods for solving matrix problems. The algorithm, although derived from integral equations, can be considered to be a special case of finite difference formalisms. A large variety of two and three-dimensional field problems can be solved by computer programs based on this approach: electrostatics and magnetostatics, lowfrequency eddy currents in solid and laminated iron cores, high-frequency modes in resonators, waves on dielectric or metallic waveguides, transient fields of antennas and waveguide transitions, transient fields of free-moving bunches of charged particles etc.

Firstly, we will explain the general method. Then we will focus on the calculation of wake potentials, cavity modes and loss parameters.

The Method. In order to avoid specializations of Maxwell's equations prior to numerical solution it is advantageous to solve Maxwell's equations directly, rather than solving a partial differential equation derived from them. Using SI units, $\mathbf{E}$ and $\mathbf{H}$ for the electric and magnetic field strength, $\mathrm{D}$ and $\mathrm{B}$ for the flux densities and $\mathbf{J}$ for the current density the equations to solve read as:

$$
\begin{gathered}
\oint_{\partial A} \mathbf{E} \cdot d \mathbf{s}=-\iint_{A} \frac{\partial \mathbf{B}}{\partial t} \cdot d \mathbf{A} \\
\oint_{\partial A} \mathbf{H} \cdot d \mathbf{s}=\iint_{A}\left(\frac{\partial \mathbf{D}}{\partial t}+\mathbf{J}\right) \cdot d \mathbf{A} \\
\iint_{V} \mathbf{B} \cdot d \mathbf{A}=0 \\
\iint_{V}\left(\frac{\partial \mathbf{D}}{\partial t}+\mathbf{J}\right) \cdot d \mathbf{A}=0
\end{gathered}
$$

with the following relations:

$$
\mathbf{D}=\epsilon \mathbf{E}, \quad \mathbf{B}=\mu \mathbf{H}, \quad \mathbf{J}=\kappa \mathbf{E}+\rho \mathbf{v} .
$$

A grid $\mathbf{G}$ is defined in the orthogonal coordinate system $\mathbf{r}=\mathbf{r}(u, v, u)$ as:

$$
\begin{aligned}
& \mathbf{G}=\left\{\left(u_{i}, v_{j}, w_{k}\right) ; \quad u_{1}<u_{i}<u_{I}, i=2, . ., I-1 ;\right. \\
& v_{1}<v_{j}<v_{J}, j=2, . ., J-1 \text {; } \\
& \left.w_{1}<w_{k}<w_{K}, k=2, . ., K-1\right\} .
\end{aligned}
$$

All nodes of $\mathrm{G}$ are numbered linearly by:

$$
\begin{gathered}
n=1+(i-1) M_{u}+(j-1) M_{v}+(k-1) M_{w}, \\
n=1, . ., N=I J I .
\end{gathered}
$$


Usually one uses $M_{u}=1, M_{v}=I, M_{u}=I J$ or any permutation of these three assignments. Figure 18 shows such a three-dimensional grid.

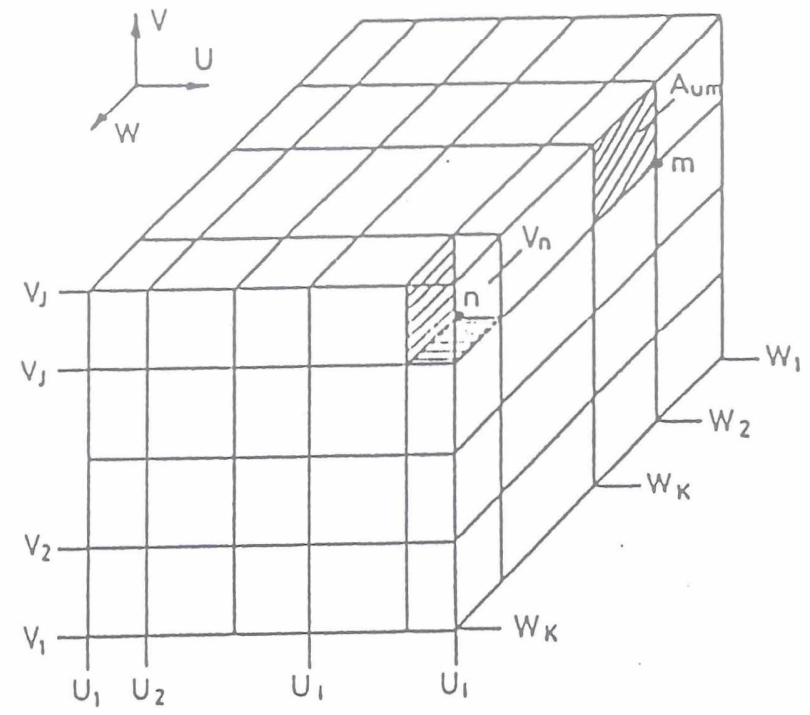

Three dimensional grid in the orthogonal not necessarily cartesian system $(\mathrm{u}, \mathrm{v}, \mathrm{w})$.

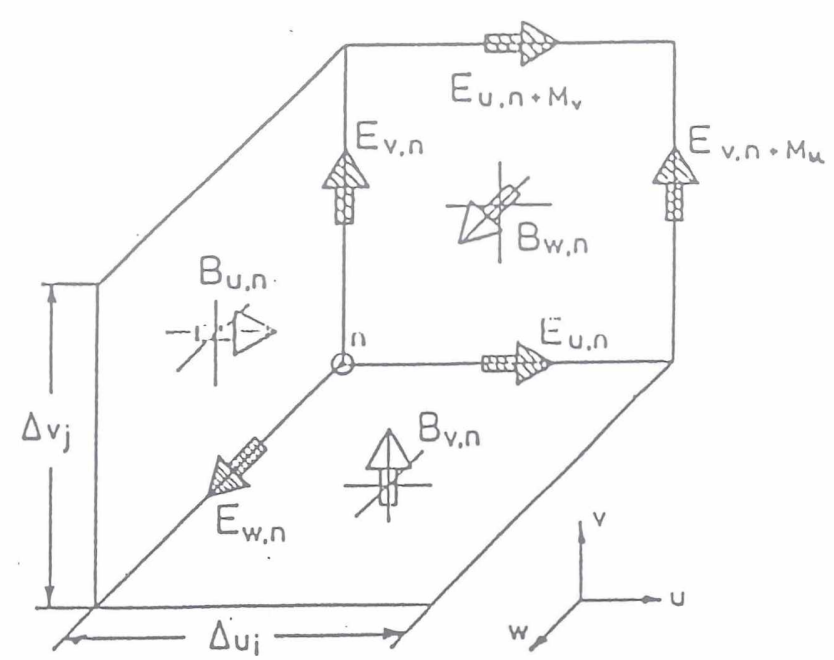

Allocation of unknown field components in the grid $\mathbf{G}$ showing the indexing.

Figure 18: Three dimensional grid used for the numerical calculations

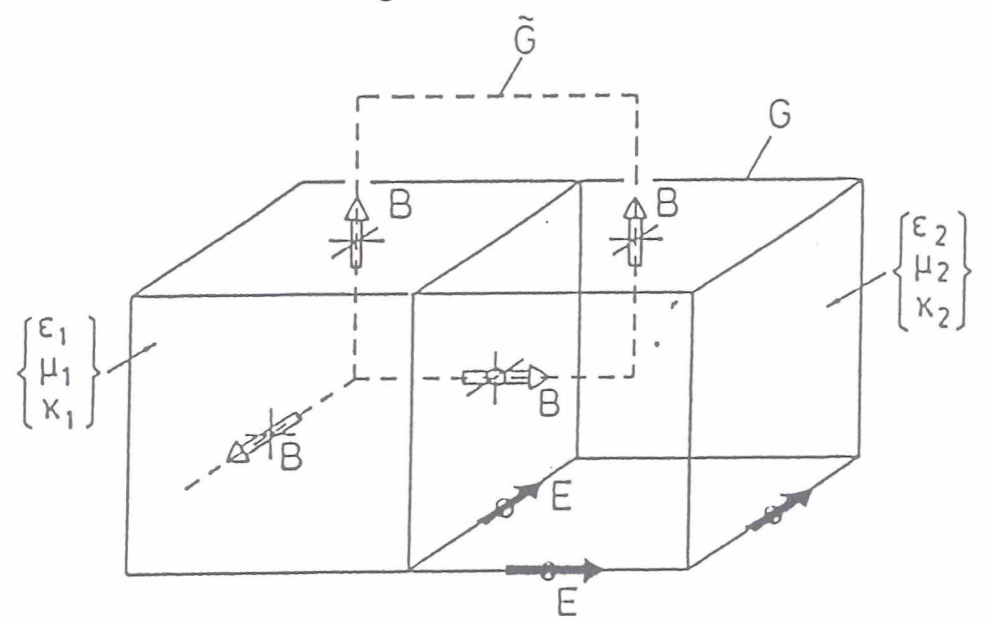

Figure 19: Two neighbouring mesh cells showing the allocation of the electric and magnetic field components in $\mathrm{G}$ and $\tilde{\mathrm{G}}$.

The functions which describe the field distribution in the volume under consideration are replaced by discrete field values in each cell of the grid. Thus one has to allocate components to grid nodes, lines, areas or elementary volumes. We will not choose the obvious allocation of calculating all six field components at the grid nodes. Such an allocation would cause serious problems at surfaces of materials where some of the field components are not continuous. Instead, the components of $\mathrm{E}$ are placed at the midpoints of the edges of the grid cells and the components of $\mathbf{B}$ at the centre of each face, 
as shown in Figure 2, extending the Yee Algorithm [6] to more general field problems $[7,8,9]$.

This kind of allocation has the distinct advantage that the transition from one cell to the next involves only continuous components, tangential $\mathbf{E}$ fields and perpendicular B fields (see Figure 19); thus Maxwell's equations are always satisfied, in this respect, even when different material fillings are involved. We now apply a first-order integration formula in order to approximate the left hand side of the first Maxwell equation and obtain the following algebraic expression replacing the integral along the border of an elementary cell:

$$
\begin{aligned}
\oint_{\partial A_{w, n}} \mathbf{E} \cdot d \mathbf{s}= & +\left(u_{i+1}-u_{i}\right) E_{u, n}+\left(v_{j+1}-v_{j}\right) E_{v, n+M_{u}} \\
& -\left(u_{i+1}-u_{i}\right) E_{u, n+M_{v}}-\left(v_{j+1}-v_{j}\right) E_{v, n} \\
& +O\left(\left(u_{i+1}-u_{i}\right)^{2}\right)+O\left(\left(v_{j+1}-v_{j}\right)^{2}\right) .
\end{aligned}
$$

For the right hand side of that equation, we obtain (again by the lowest-order integration formula):

$$
\iint_{A_{w, n}} \frac{\partial \mathbf{B}}{\partial t} \cdot d \mathbf{A}=\dot{\mathbf{B}}_{w, n}\left(u_{i+1}-u_{i}\right)\left(v_{j+1}-v_{j}\right)+O\left(\left(\left(u_{i+1}-u_{i}\right)\left(v_{j+1}-v_{j}\right)\right)^{2}\right) .
$$

Equating the two expressions yields a discrete replacement for the first Maxwell equation on each surface of the grid cells and thus on every area composed of mesh cell surfaces. In order to describe all these equations for all surfaces we introduce a basic discretization matrix with only two bands and with elements taking only the values 0 , +1 or -1 :

$$
\mathbf{P}_{\mathbf{u}}:=\left(\mathbf{P}_{\mathbf{u}}\right)_{m, p}=\left\{\begin{aligned}
-1 & ; p=m \\
+1 & ; p=m+M_{u} \quad{ }_{m, p=1, \ldots, N} . \\
0 & ; \text { else }
\end{aligned}\right.
$$

Out of such simple matrices we combine the matrix $\mathbf{C}$ that replaces the contour integral operator in the first Maxwell equation for all mesh points:

$$
\mathbf{C}=\left(\begin{array}{ccc}
\mathbf{0} & -\mathbf{P}_{w} & \mathbf{P}_{v} \\
\mathbf{P}_{w} & 0 & -\mathbf{P}_{u} \\
-\mathbf{P}_{v} & \mathbf{P}_{u} & 0
\end{array}\right)
$$

All unknown components of the electric field $\mathbf{E}$ are put into a vector of dimension $3 \mathrm{~N}=3(\mathrm{IJK}):$

$$
\mathbf{e}=\left(E_{u, 1}, E_{u, 2}, \ldots, E_{w, N}\right)^{t}
$$

and we replace $\mathbf{D}$ by $\mathbf{d}, \mathbf{B}$ by $\mathbf{b}, \mathbf{H}$ by $\mathbf{h}$ and $\mathbf{J}$ by $\mathbf{j}$ respectively. The topological part of the contour integral (curl) is represented by the matrix $\mathbf{C}$. The actual length of the integration path is put into a diagonal matrix:

$$
\mathbf{D}_{\mathrm{s}}=\operatorname{Diag}\left(\Delta u_{1}, . ., \Delta u_{N}, \Delta v_{1}, . ., \Delta v_{N}, \Delta w_{1}, . ., \Delta w_{N}\right),
$$


$\Delta u_{n} \quad \Delta v_{n}$ and $\Delta w_{n}$ are the mesh step sizes. The surfaces of the grid cells compose the diagonal matrix:

$$
\begin{gathered}
\mathbf{D}_{\mathbf{A}}=\operatorname{Diag}\left(A_{u, 1}, \ldots A_{u, N}, A_{v, 1}, . ., A_{v, N}, A_{w, 1}, . ., A_{w, N}\right), \\
A_{u, n}=\Delta v_{n} \Delta w_{n}, \quad A_{v, n}=\Delta w_{n} \Delta u_{n}, \quad A_{w, n}=\Delta u_{n} \Delta v_{n} .
\end{gathered}
$$

Finally we can give a matrix equation using all these definitions that replaces the first. Maxwell equation on all cell surfaces:

$$
\mathrm{CD}_{\mathrm{s}} \mathrm{e}=-\mathrm{D}_{\mathrm{A}} \dot{\mathrm{b}}
$$

The special allocation of field components in the grid generates a second, dual grid $\tilde{\mathbf{G}}$ in which the magnetic flux densities are allocated as are the electric field components in the original grid $\mathrm{G}$, see Figure 19 .

The dual grid is defined by:

$$
\begin{aligned}
& \tilde{\mathbf{G}}=\left\{\left(\tilde{u}_{i}, \tilde{v}_{j}, \tilde{w}_{k}\right) ; \quad \tilde{u}_{1}<\tilde{u}_{i}<\tilde{u}_{I}, i=2, . ., I-1 ;\right. \\
& \tilde{v}_{1}<\tilde{v}_{j}<\tilde{v}_{J}, j=2, . ., J-1 \text {; } \\
& \left.\tilde{w}_{1}<\tilde{w}_{k}<\tilde{w}_{K}, k=2, . ., K-1\right\} \text {, } \\
& \tilde{u}_{i}=\left(u_{i+1}+u_{i}\right) / 2 ; 1 \leq i \leq I-1 ; \tilde{u}_{I}=0, \\
& \tilde{v}_{j}=\left(v_{j+1}+v_{j}\right) / 2 ; 1 \leq j \leq J-1 ; \tilde{v}_{J}=0 \text {, } \\
& \tilde{w}_{k}=\left(w_{k+1}+w_{k}\right) / 2 ; 1 \leq k \leq K-1 ; \tilde{w}_{K}=0 \text {. }
\end{aligned}
$$

We can also define matrices that hold the mesh step sizes and cell areas as for the original grid $\mathrm{G}$ :

$$
\begin{gathered}
\tilde{\mathbf{D}}_{\mathrm{s}}=\operatorname{Diag}\left(\tilde{\Delta} u_{1}, . ., \tilde{\Delta} u_{N}, \tilde{\Delta} v_{1}, . ., \tilde{\Delta} v_{N}, \tilde{\Delta} w_{1}, . ., \tilde{\Delta} w_{N}\right), \\
\tilde{\mathbf{D}}_{\mathbf{A}}=\operatorname{Diag}\left(\tilde{A}_{u, 1}, . ., \tilde{A}_{u, N}, \tilde{A}_{v, 1}, . ., \tilde{A}_{v, N}, \tilde{A}_{w, 1}, . ., \tilde{A}_{w, N}\right), \\
\tilde{A}_{u, n}=\tilde{\Delta} v_{n} \tilde{\Delta} w_{n}, \quad \tilde{A}_{v, n}=\tilde{\Delta} w_{n} \tilde{\Delta} u_{n}, \quad \tilde{A}_{w, n}=\tilde{\Delta} u_{n} \tilde{\Delta} v_{n} .
\end{gathered}
$$

When solving the second Maxwell equation in a way similar to the first one, we have to face the fact that the magnetic field $\mathbf{H}$ is not defined and thus has to be calculated from the flux density piecewise along the integration path. Also, the integration over the electric flux density is no longer a simple product of an area with a component but the sum over four parts on each of which the flux has to be determined from the defined $\mathrm{E}$ and the material constants. Finally we obtain:

$$
\tilde{\mathbf{C}} \tilde{\mathbf{D}}_{\mathrm{s}} \mathbf{D}_{\mu}^{-1} \mathbf{b}=\tilde{\mathbf{D}}_{\mathrm{A}}(\dot{\mathrm{d}}+\mathbf{j})
$$

Note that the contour integral operator on the dual grid, $\tilde{\mathbf{C}},(\mathbf{c u r l})$ is related to $\mathbf{C}$ simply by

$$
\tilde{\mathbf{C}}=\mathbf{C}^{\mathrm{t}} \text {. }
$$


The diagonal matrices which take care of the piecewise different values for the material constants are:

$$
\mathbf{D}_{\mu}, \quad \tilde{\mathbf{D}}_{\epsilon}, \text { and } \tilde{\mathbf{D}}_{\kappa} .
$$

Note that $\mu, \epsilon$ and $\kappa$ may have different properties in the directions $u, v$ and $w$. Although not originally defined in the grid, one may relate the field strength, flux density and current density and formally define a magnetic field and an electric flux density by:

$$
\begin{aligned}
\mathbf{b}=\mathbf{D}_{\mu} \mathbf{h}, & (\mathbf{B}=\mu \mathbf{H}), \\
\mathbf{d}=\tilde{\mathbf{D}}_{\epsilon} \mathbf{e}, & (\mathbf{D}=\epsilon \mathbf{E}), \\
\mathbf{j}=\tilde{\mathbf{D}}_{\kappa} \mathbf{e}+\mathbf{D}_{\rho} \mathbf{v}, & (\mathbf{J}=\kappa \mathbf{E}+\rho \mathbf{v}) .
\end{aligned}
$$

In order to complete the transformation of Maxwell's equations to the grid space, we approximate the divergence equation by integrating $\mathbf{B}$ over surfaces of each mesh cell of $\mathbf{G}$. We can then write this equation by defining the discrete div operator on $\mathbf{G}$ as:

$$
\mathbf{S}=\left(\mathbf{P}_{\mathrm{u}}\left|\mathbf{P}_{\mathrm{v}}\right| \mathbf{P}_{\mathrm{w}}\right)
$$

and obtain:

$$
\mathrm{SD}_{\mathrm{A}} \mathrm{b}=0
$$

This equation allocates the (non-existent) magnetic charges to nodes of $\tilde{G}$. The corresponding matrix on the dual grid $\tilde{\mathbf{G}}$ is simply given by:

$$
\tilde{\mathrm{S}}=\left(\mathbf{P}_{\mathrm{u}}^{\mathrm{t}}\left|\mathbf{P}_{\mathrm{v}}^{\mathrm{t}}\right| \mathbf{P}_{\mathrm{w}}^{\mathrm{t}}\right) \text {. }
$$

So the continuity equation in the grid space reads as:

$$
\tilde{\mathbf{S}} \tilde{\mathbf{D}}_{\mathrm{A}}(\dot{\mathrm{d}}+\mathbf{j})=0
$$

Thus electric charges are defined on nodes of the original grid $\mathbf{G}$.

Properties of the grid equations. One of the outstanding properties of Maxwell's grid equations is that properties of analytical solutions have their exact analogues in the grid space. The analytical identity div curl $\equiv 0$ reads in the grid space as:

$$
\operatorname{div} \text { curl } \equiv 0 \leftrightarrow \tilde{\mathrm{S}} \tilde{\mathrm{C}} \equiv \mathrm{SC} \equiv 0
$$

The analytical property that scalar potential fields are curl free is also found in the grid space as:

$$
\text { curl grad } \equiv 0 \Leftrightarrow \mathrm{C}^{t} \mathrm{~S}^{\mathrm{t}} \equiv \tilde{\mathrm{C}} \tilde{\mathrm{S}}^{\mathrm{t}} \equiv 0 .
$$

These properties of the Maxwell grid equations not only offer a unique tool to test. numerical results for their physical correctness but also avoid the occurrence of incorrect. solutions in the calculation of three-dimensional eigenmodes in resonators, or at least. their identification [8]. This is quite important since numerical errors in the matrix 
algorithms may occur and cannot generally be detected. In the field of accelerator physics, one often investigates unknown phenomena by using such codes, so that an incorrect solution could lead to physical misinterpretations.

The Maxwell Grid Equations. Without specifying anything about the shape of materials nor the time dependence of the fields, we have obtained a set of matrix equations that approximate Maxwell's equations on a double grid $\{\mathbf{G}, \tilde{\mathbf{G}}\}\left(\mathbf{R}^{3}\right.$ and $\mathbf{R}^{3 N}$ represent the physical spaces and $\mathbf{R}_{+}$the time):

$$
\begin{aligned}
& \text { Real Space } \quad \Leftrightarrow \quad \text { Grid Space } \\
& \mathbf{R}^{3} \otimes \mathbf{R}_{+} \quad \mathbf{R}^{3 N} \otimes \mathbf{R}_{+} \\
& \oint_{\partial A} \mathbf{E} \cdot d \mathbf{s}=-\iint_{A} \frac{\partial \mathbf{B}}{\partial t} \cdot d \mathbf{A} \Leftrightarrow \quad \mathbf{C D}_{\mathbf{s}} \mathbf{e}=-\mathbf{D}_{\mathbf{A}} \dot{\mathbf{b}} \\
& \oint_{\partial A} \mathbf{H} \cdot d \mathbf{s}=\iint_{A}\left(\frac{\partial \mathbf{D}}{\partial t}+\mathbf{J}\right) \cdot d \mathbf{A} \Leftrightarrow \quad \tilde{\mathbf{C}} \tilde{\mathbf{D}}_{\mathbf{s}} \mathbf{h}=\tilde{\mathbf{D}}_{\mathbf{A}}(\dot{\mathbf{d}}+\mathbf{j}) \\
& \iint_{V} \mathbf{B} \cdot d \mathbf{A}=\mathbf{0} \Leftrightarrow \quad \mathrm{SD}_{\mathbf{A}} \mathrm{b}=0 \\
& \iint_{V}\left(\frac{\partial \mathbf{D}}{\partial t}+\mathbf{J}\right) \cdot d \mathbf{A}=\mathbf{0} \Leftrightarrow \quad \tilde{\mathbf{S}} \tilde{\mathbf{D}}_{\mathbf{A}}(\dot{\mathbf{d}}+\mathbf{j})=\mathbf{0} \\
& \mathrm{D}=\epsilon \mathbf{E} \Leftrightarrow \quad \mathrm{d}=\tilde{\mathbf{D}}_{\epsilon} \mathbf{e} \\
& \mathbf{B}=\mu \mathrm{H} \Leftrightarrow \quad \mathbf{b}=\mathbb{D}_{\mu} \mathbf{h} \\
& \mathbf{J}=\kappa \mathbf{E}+\rho \mathbf{v} \Leftrightarrow \quad \mathbf{j}=\tilde{\mathbf{D}}_{\kappa} \mathbf{e}+\mathbf{D}_{\rho} \mathbf{v} \\
& \text { div curl } \equiv 0 \Leftrightarrow \quad \mathrm{S} \mathrm{C}=\tilde{\mathrm{S}} \tilde{\mathrm{C}}=0 \\
& \text { curl grad } \equiv 0 \Leftrightarrow \quad \tilde{\mathrm{C}}^{\mathrm{t}} \tilde{\mathrm{S}}^{\mathrm{t}}=\mathrm{C}^{\mathrm{t}} \mathrm{S}^{\mathrm{t}}=0
\end{aligned}
$$

\subsubsection{Short and Long Range Wakes}

In order to solve these equations one has only to perform matrix manipulations and to solve the established matrix problem numerically.

Short range wake fields can be calculated efficiently in the time domain. For transient fields with a central time difference formula, step size $\delta t$, no currents and no losses, one obtains the Yee-algorithm [6]. In the presence of free moving charges the algorithm has more constraints to ensure charge conservation [10]. The upper index n denotes the time in units of $\delta t$. These recursive formulae allow the easy calculation of transient fields of antennas, particle beams in accelerators [10] or wave propagation problems even in the lossy case:

$$
\begin{aligned}
\mathbf{b}^{n+1} & =\mathbf{b}^{n}-\Delta t \mathbf{D}_{A}^{-1} \mathbf{C D}_{s} \mathbf{e}^{n+0.5} \\
\mathbf{e}^{n+1.5} & =\mathbf{D}_{a} \mathbf{e}^{n+0.5}+\left(1-\mathbf{D}_{a}\right) \tilde{\mathbf{D}}_{\kappa}^{-1} \tilde{\mathbf{D}}_{A}^{-1} \tilde{\mathbf{C}} \tilde{\mathbf{D}}_{s} \mathbf{D}_{\mu}^{-1} \mathbf{b}^{n+1}-\left(1-\mathbf{D}_{a}\right) \tilde{\mathbf{D}}_{\kappa}^{-1} j_{c}^{n+1}
\end{aligned}
$$


with

$$
\mathbf{D}_{a}=\exp \left(-\tilde{\mathbf{D}}_{\varepsilon}^{-1} \mathbf{D}_{\kappa} \Delta t\right)
$$

For detailed setting up of the final matrix problem refer to the literature $[8,9]$. Knowing the electric and magnetic fields for each time step it is possible to obtain the wake potential directly by a numerical integration of

$$
\boldsymbol{W}(x, y, s)=\frac{1}{q} \int_{-\infty}^{\infty} d z\left[\boldsymbol{E}(x, y, z, t)+c e_{z} \times B(x, y, z, t)\right]_{t=(s+z) / c}
$$

if the charge $q$ moves along the $z$-axis.

As mentioned in section 2.5 it is advantageous to perform the integration for all positions $(x, y)$ at the side tubes because the integration interval is then finite. The longitudinal wake potential for any position $(x, y)$ is then obtained as a solution of the equation

$$
\nabla_{\perp}^{2} W_{\|}(x, y, s)=0
$$

and the transverse one by an application of Panofsky-Wenzel-Theorem.

The long range wake potential is dominated by the interaction of the beam with some cavity modes. For arbitrarily shaped cavities the eigen modes have to be calculated numerically. The electric fields simultaneously have to obey the following two equations, that can be directly derived from Maxwell's equations

$$
\begin{aligned}
\operatorname{curl} \frac{1}{\mu} \operatorname{curl} \mathbf{E} & =\omega^{2} \epsilon \mathbf{E} \\
\operatorname{div} \epsilon \mathbf{E} & =0
\end{aligned}
$$

The numerical formulation can be derived again by matrix manipulation:

$$
\begin{gathered}
\left.\tilde{\mathbf{C}} \tilde{\mathbf{D}}_{\mathrm{s}} \mathrm{D}_{\mu}^{-1} \mathrm{D}_{\mathrm{A}}^{-1} \mathrm{C} \mathrm{D}_{\mathrm{s}}\right) \mathrm{e}=\omega^{2} \tilde{\mathbf{D}}_{\mathrm{A}} \tilde{\mathbf{D}}_{\epsilon} \mathrm{e} \\
\tilde{\mathbf{S}} \tilde{\mathbf{D}}_{\mathrm{A}} \tilde{\mathbf{D}}_{\epsilon} \mathrm{e}=0
\end{gathered}
$$

In order to convert the algorithm to a symmetric form we must perform a diagonal transformation, which physically replaces the field strength by the root of the local energy density (except from a scalar factor):

$$
\mathbf{e}^{\prime}=\left(\tilde{\mathbf{D}}_{\epsilon} \tilde{\mathbf{D}}_{\mathbf{A}} \mathbf{D}_{\mathbf{s}}\right)^{1 / 2} \mathbf{e}
$$

One obtains an $3 \mathrm{~N} \times 3 \mathrm{~N}$ eigenvalue equation, the eigenvalues of which are the resonant frequencies squared [8]:

$$
(\tilde{\mathrm{D}} \tilde{\mathrm{C}} \mathrm{D})(\tilde{\mathrm{D}} \tilde{\mathrm{C}} \mathrm{D})^{t} \mathrm{e}^{\prime}=\omega^{2} \mathrm{e}^{\prime} .
$$

With the matrices

$$
\mathbf{D}=\left(\mathbf{D}_{\mathbf{A}}^{-1} \tilde{\mathbf{D}}_{\mathbf{s}} \mathbf{D}_{\mu}^{-1}\right)^{1 / 2},
$$




$$
\tilde{\mathbf{D}}=\left(\tilde{\mathbf{D}}_{\mathbf{A}}^{-1} \mathbf{D}_{\mathbf{s}} \tilde{\mathbf{D}}_{\epsilon}^{-1}\right)^{1 / 2}
$$

The resulting matrix has a multiply vanishing eigenvalue for $\omega=0$ as the existence of static solutions cannot be excluded by curl operations only. This solutions can be identified easily by resubstituting the eigenvectors in the div equation.

The analytical analogue to this combination of equations is the equation curl curlE = grad $\operatorname{div} \mathbf{E}-\nabla^{2} \mathbf{E}$ and $\operatorname{div} \mathbf{E}=0$ thus solving $\nabla^{2} \mathbf{E}$.

After an numerical solution for the cavity modes the loss parameter $k$ can be easily calculated from the voltage

$$
V=\int d z E_{z}(\boldsymbol{r}) \exp (i \omega z / c)
$$

and the total energy stored in the specific mode:

$$
U=\frac{\epsilon_{0}}{2} \int d^{3} r|\boldsymbol{E}(\boldsymbol{r})|^{2} .
$$

(See also section 3.2). 


\subsection{Examples}

The in the preceding sections presented method to solve the Maxwell grid equations in two and three dimensions is implemented in the CAD-program packet MAFIA [11]. We want to present here some calculations obtained with this program, TBCI or URMEL, which are now included into the newest release of MAFIA.

The acronym MAFIA stands for the solution of MAxwell's equations by the Finite Integration Algorithm. A short description of the modules is given in the next table.

\begin{tabular}{|c|c|c|}
\hline Module & Description & Special Features \\
\hline $\mathrm{M}$ & $\begin{array}{l}\text { mesh generator, trans- } \\
\text { lates the physical prob- } \\
\text { lem into mesh data }\end{array}$ & $\begin{array}{l}\text { many predefined shapes, interactive } \\
\text { remeshing }\end{array}$ \\
\hline S & $\begin{array}{l}\text { static solver, electro- } \\
\text { and magnetostatic prob- } \\
\text { lems }\end{array}$ & $\begin{array}{l}\text { open boundary, nonlinear magnetic } \\
\text { material properties, advanced multi- } \\
\text { grid solver for very large meshes }\end{array}$ \\
\hline $\mathrm{R}$ & $\begin{array}{l}\text { matrix generator, eigen- } \\
\text { value solver for res- } \\
\text { onator and waveguide } \\
\text { problems }\end{array}$ & $\begin{array}{l}\text { periodic boundary conditions, reso- } \\
\text { nance frequencies (several hundred } \\
\text { modes), waveguide modes and propa- } \\
\text { gation parameters }\end{array}$ \\
\hline $\begin{array}{l}\mathrm{T} 2 \\
\mathrm{~T} 3\end{array}$ & time domain solver & $\begin{array}{l}\text { losses, open boundary, stimulation by: } \\
\text { initial field, current, waveguide, inci- } \\
\text { dent waves; wake potentials }\end{array}$ \\
\hline $\begin{array}{l}\text { TS2 } \\
\text { TS3 }\end{array}$ & $\begin{array}{l}\text { particle in cell code, cal- } \\
\text { culates equation of mo- } \\
\text { tion for free moving } \\
\text { charges }\end{array}$ & $\begin{array}{l}\text { losses, stimulation by initial field, } \\
\text { calculation of trajectories, velocities, } \\
\text { charge densities etc. }\end{array}$ \\
\hline W3 & $\begin{array}{l}\text { eddy current solver, fre- } \\
\text { quency domain }\end{array}$ & losses, current stimulation \\
\hline $\mathbf{P}$ & $\begin{array}{l}\text { post processor, displays } \\
\text { results and calculates } \\
\text { secondary field quanti- } \\
\text { ties }\end{array}$ & $\begin{array}{l}1,2 \text { and } 3-D \text { graphics of scalar and } \\
\text { vector fields, power loss integrals, } \\
\text { field energy, far field pattern, shunt } \\
\text { impedance }\end{array}$ \\
\hline
\end{tabular}

Figure 20 shows the interconnections between the various programs of the third release.

The first example is a radiofrequency quadrupole ( $R F Q$ ) that has been calculated with the MAFIA frequency domain module $\mathrm{R} / \mathrm{E}$. The device might be tuned for a certain minimum eigenmode or in order to minimize the losses. Three dimensional calculations are important especially for the end region of such a RFQ. A careful design using the MAFIA mesh generator has been carried out, using about 75,000 unknowns. 
The lowest eigenmode calculated, $344.78 \mathrm{MHz}$ is in good agreement with measurements and other calculations. Figure 21 displays the power loss. This loss is strongest on the outside wall. The power loss can be calculated by the MAFIA-postprocessor from the field solutions.

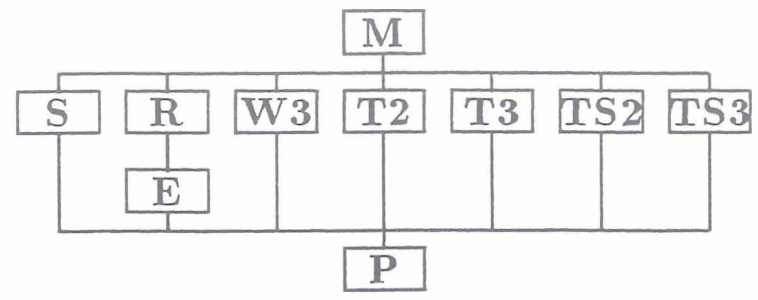

Figure 20: : The MAFIA System with its Inter-relationships.

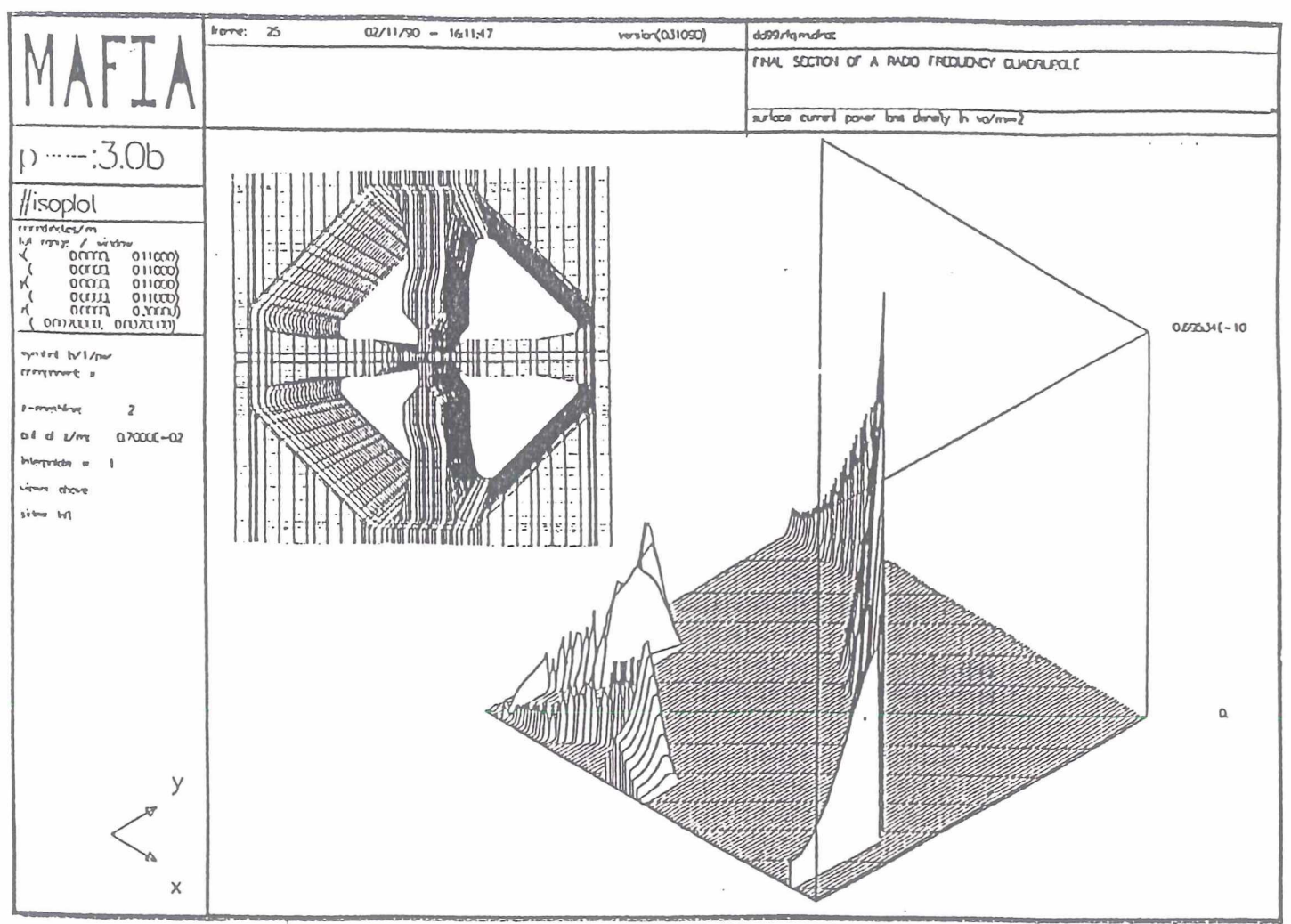

Figure 21: RFQ: The geometry and the power loss is shown. 
The figure 22 shows the geometry of a kicker magnet calculated for the GSI with MAFIA. With the modules $\mathrm{R}$ and $\mathrm{E}$ the modes of the structures have been calculated in order to evaluate the loss parameters.

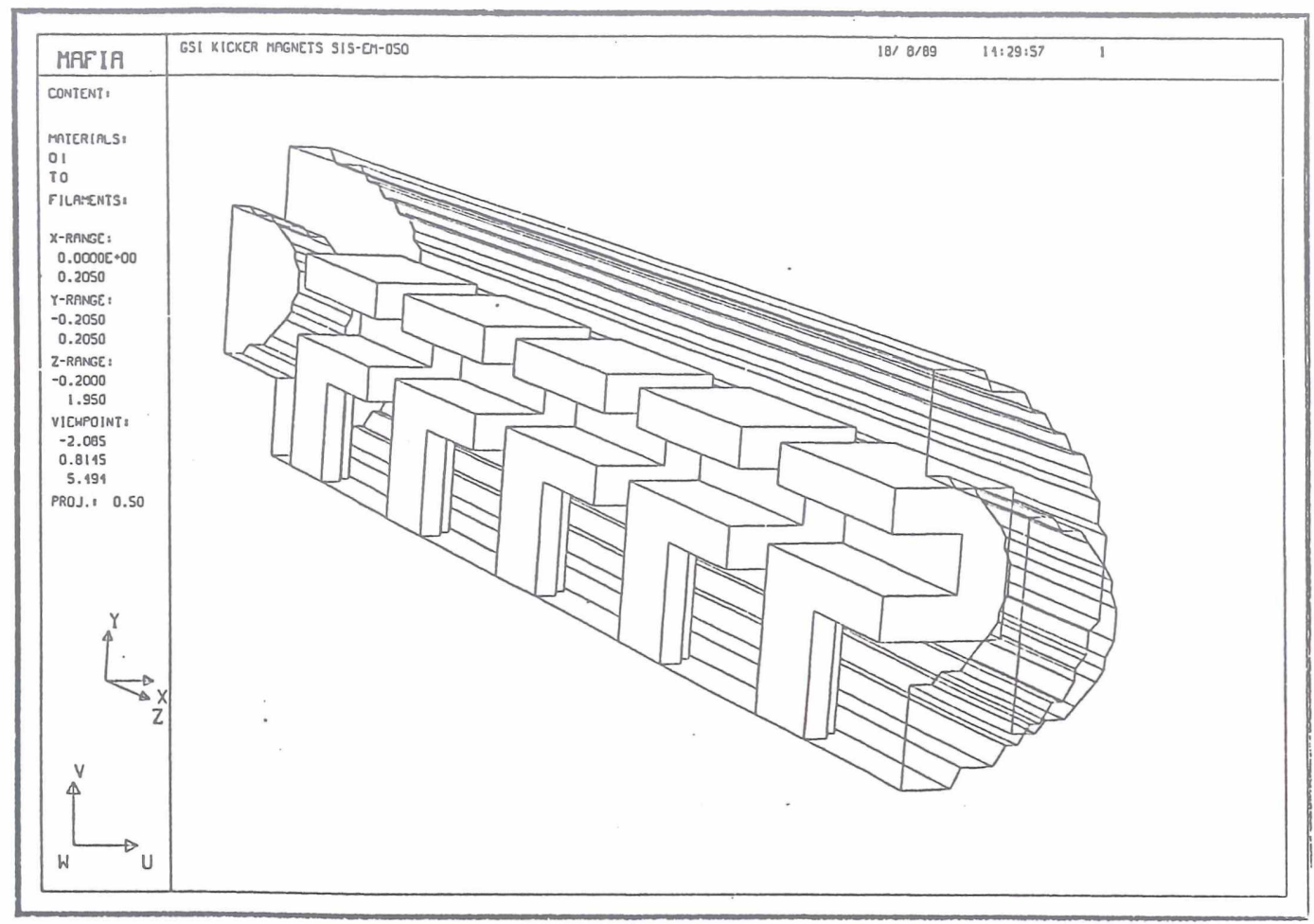

Figure 22: The geometry of a kicker magnet is shown

The next two examples show wake field calculations with the computer code TBCI. A Gaussian bunch traverses a bellow structure (figure 23) and a PETRA cavity (figure 24) respectively. 

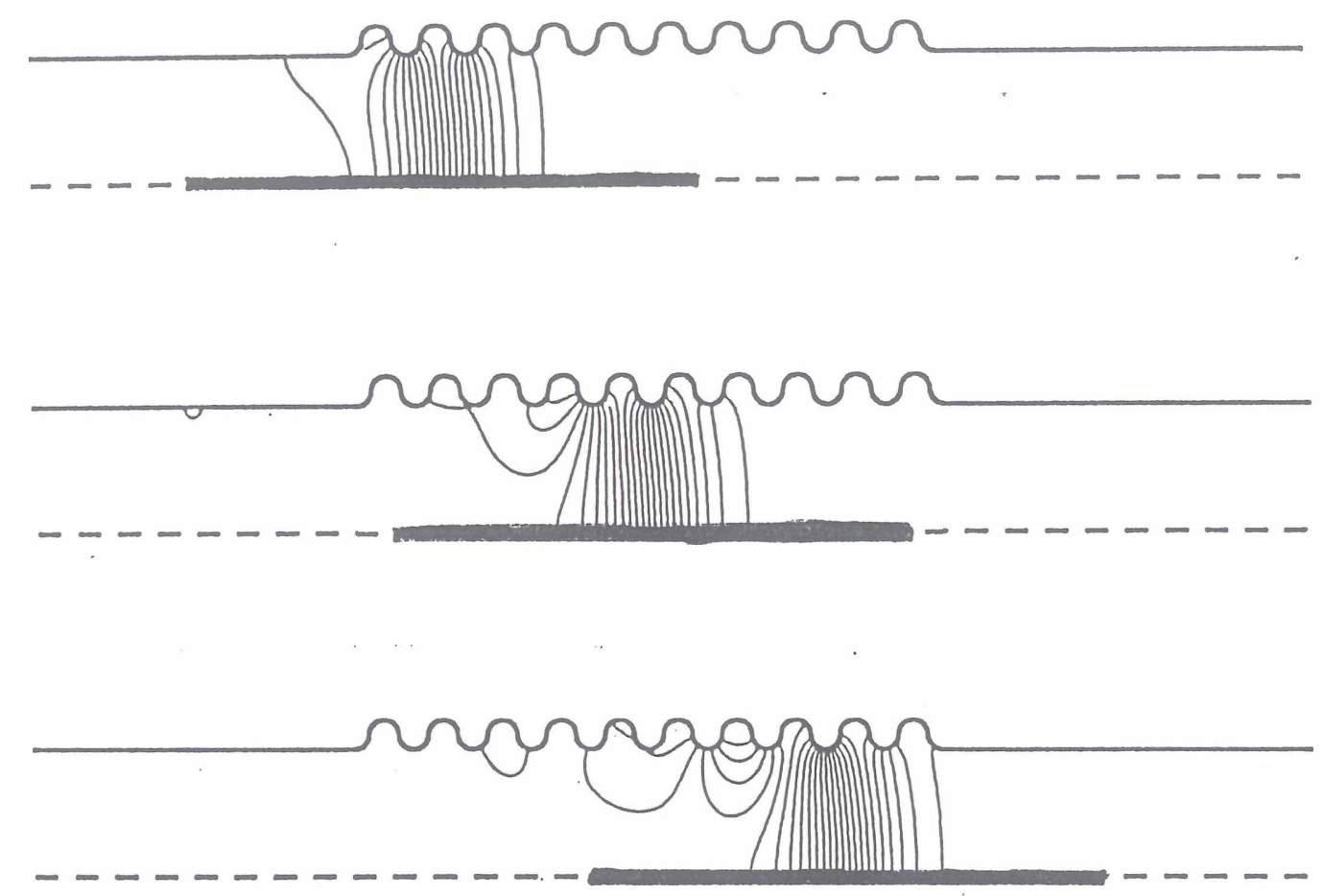

Figure 23: Wake fields in a bellow structure. The bar represents the bunch. The electric field is represented by lines of constant $\int d t r H_{\varphi} \sim r|E|$ 

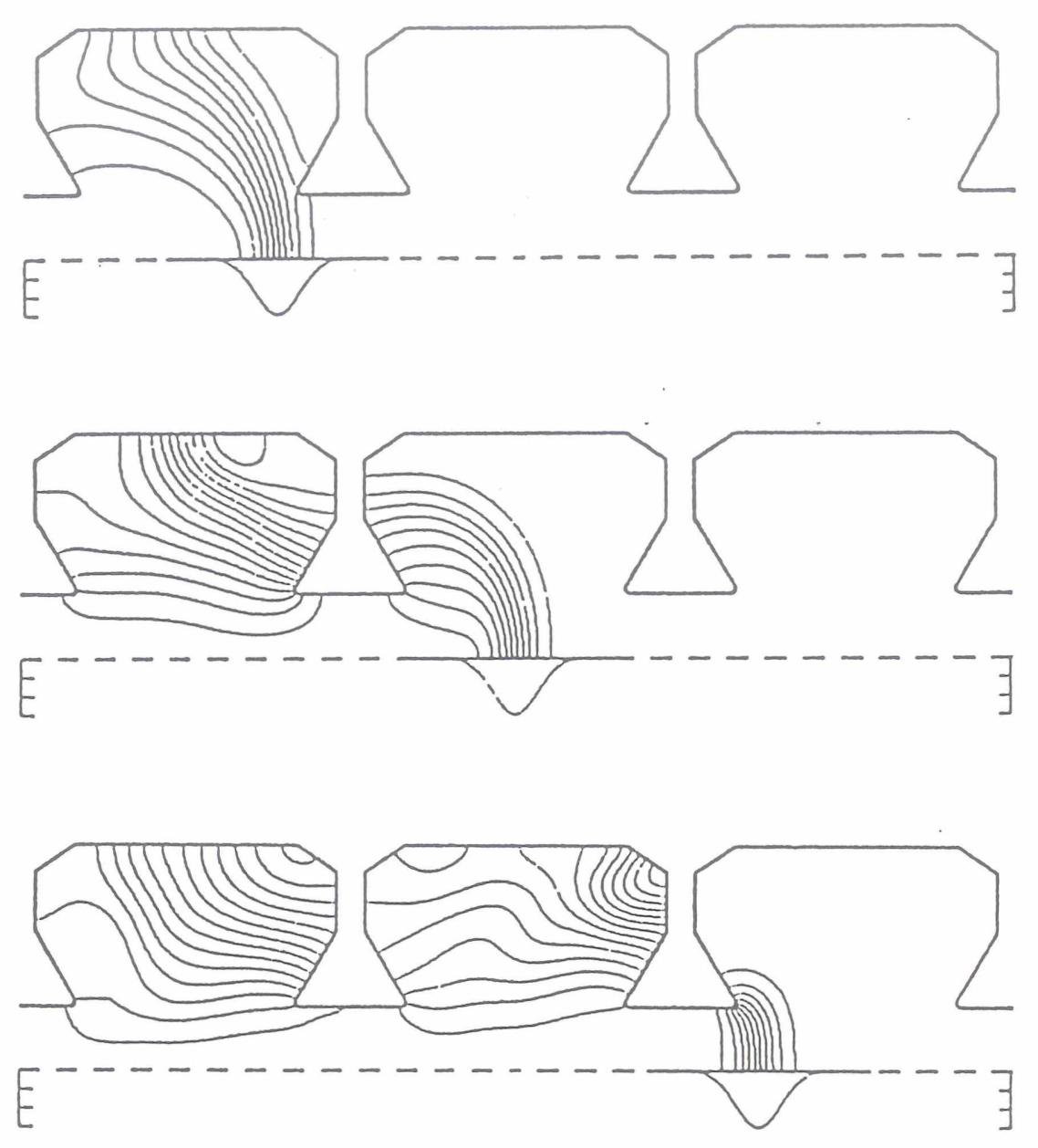

Figure 24: Wake fields in a PETRA cavity generated by a Gaussian bunch. The electric field is represented by lines of constant $\int d t r H_{\varphi} \sim r|E|$ 


\section{Effects of Wakes and Impedances}

\subsection{Heating}

Due to wake fields the bunch lost energy which heats the traversed structure. We may distinguish heating effects due to short range wakes and due to long range wakes. Short range wake field effects are single turn effects in a storage ring. The heating due to the instantaneous energy loss cause normally no problems for structures with a good thermal conductivity. Long range wake field effects are important if a single mode is driven resonantly by successive turns of the bunches. All effects can be calculated from the loss parameters. We will give for each of the heating effects an example.

Transient effects. Consider a storage ring characterized by the

- revolution time $T_{0}=7.68 \mu \mathrm{s}$,

- number of bunches $N_{b}=4$,

- charge per bunch $q_{b}=40 \mathrm{nC}$.

The heating of a PETRA cavity ( 5 cells) due to transient losses can be calculated from the parasitic loss parameter

$$
k_{\text {par }}(\sigma)=1.5 \times 10^{12} \frac{\mathrm{V}}{\mathrm{As}}
$$

for a Gaussian bunch with rms length of $\sigma=1 \mathrm{~cm}$. The Heating power is given by:

$$
P=N_{b} q_{b}^{2} k_{p a r} \frac{1}{T_{0}} \approx 1250 \mathrm{~W}
$$

This heating power cause no problems for a large copper structure like a PETRA cavity.

Resonant effects. We now consider the heating due to the resonant excitation of one mode characterized by the loss parameter $k$, the revolution frequency $\omega$ and the quality factor $Q$. Note that $k$ and $\omega$ are determinated by the geometrical shape while $Q$ depends on the material properties. In section 3.4 we calculated the induced voltage if one mode is driven resonantly by the beam. We obtained for the cavity voltage

$$
V=R I \quad \text { with } R=\frac{4 k Q}{\omega}
$$

$R$ is the shunt impedance (US definition) and $I$ the current in the storage ring.

For $I=160 \mathrm{~mA}\left(N_{b}=210\right.$ bunches in HERA with $\left.q_{b}=16 \mathrm{nC}\right)$ and a shunt impedance of $R=1 \mathrm{M} \Omega$ one obtains a heating power of

$$
P=R I^{2} \approx 25 \mathrm{~kW}
$$

if in the worst case all bunches are in phase with the mode.

Any accelerator component has a finite shunt impedance not only cavities. For high currents (e.g. B-factories with $I \approx 1 \mathrm{~A}$ ) even from small shunt impedances high heating powers may result if such a mode is driven resonantly. 


\subsection{Instabilities}

As already mentioned in the introduction this section can only give a brief overview about the subject. For a more detailed treatment of instabilities we refer to the lecture of these accelerator course by A. Hofmann and the literature.

We can again distinguish short range wake field effects within one bunch and long range wake fields effects from bunch t.o bunch. Furthermore longitudinal and transverse effects on the beam can be considered. In this section we will focus on single bunch effects, especially on bunch lengthening. It is the goal to explain how the wake potential can be included into tracking calculation leading to a quantitatively prediction of bunch lengthening.

The electromagnetic interaction between a bunch and its environment can be described by a Greens's function, the $\delta$-function wake potential. This potential gives the energy loss of a witness charge behind a leading point charge after the passage of the entire structure as a function of their distant. Such $\delta$-function wake potentials can not calculated exactly for realistic structure. An approximation can be obtained by mode analysis with additionally analytical extentions.

But it is not necessary to know the $\delta$-function wake potential. An approximate Green's function can be calculated by means of the computer code TBCI. Instead of a leading point charge a leading short Gaussian bunch (super particle) is used. This is a good approximation if the rms length of the super particles are small compared to the total rms bunch length. (For a detailed analysis of the errors see [12].)

The longitudinal equations of motion are:

$$
\begin{aligned}
& \dot{\tau}=\alpha \frac{\epsilon}{E_{0}} \\
& \dot{\epsilon}=e V_{r f} \sin \left(\omega_{r f} \tau\right)
\end{aligned}
$$

$\tau$ is the particle position in time with origin at zero of the rf voltage.

$\epsilon$ is the particle energy deviation from the nominal energy $E_{0}$.

$V_{r f}$ is the rf voltage and $\alpha$ the momentum compaction.

Longitudinal wake field effects can be simply included:

$$
\dot{\epsilon}=e V_{r f} \sin \left(\omega_{r f} \tau\right)-e w(\tau)
$$

$w(\tau)$ is the wake potential at position $\tau$ which can be computed by summing all contributions of the particles in front of $\tau$ :

$$
w(\tau)=\frac{q}{N} \sum_{i=1, \tau_{2}>\tau}^{N} w_{s}\left(\tau_{i}-\tau\right)
$$

$q$ is the total charge in the bunch,

$N$ the number of super particles, and 
$w_{s}$ is the wake potential of one super particle calculated numerically by TBCI. For practical tracking calculations the super particles can be filled into bins to speed up to evaluation of the sums (see [12]).

Radiation losses and quantum excitations can be also included into the tracking equations. Figure 25 show the predicted and measured bunch length as a function of single bunch current. The good agreement of the calculations with the measurements demonstrates the usefulness of the method.

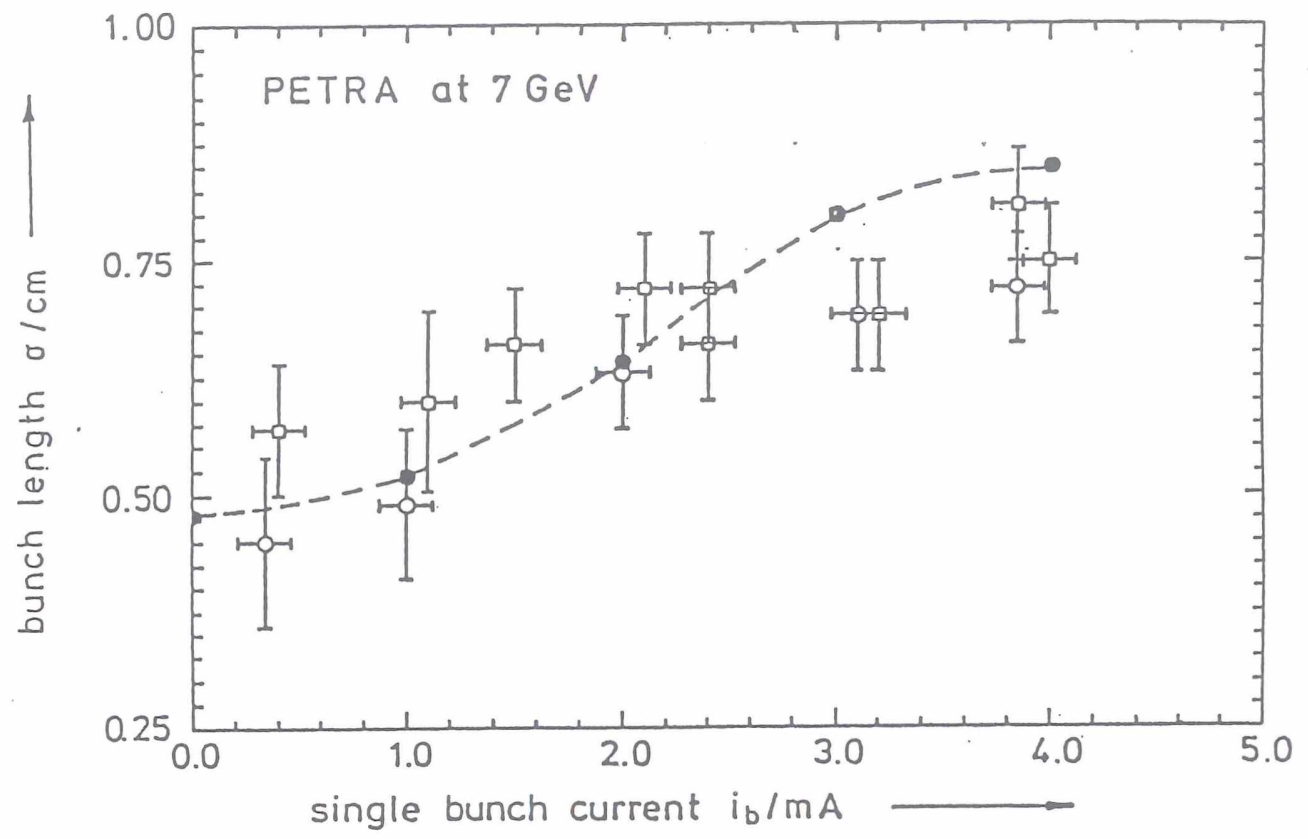

Figure 25: Bunch length as a function of the single bunch current at a energy of $7 \mathrm{GeV}$ in PETRA. The dashed line represent the result of tracking calculations.

The transverse wake field effects can studied by tracking calculations, too. Again super particles can be used to calculate the wake potential. Every super particle is kicked by the leading particles. While different approaches agreed under each other well between the simulation and the reality remained a factor of 3 to 4 . The reason was that the transverse impedance outside the cavities from separator tanks, bellows and vacuum chambers joints were not included.

A rough comparison of the transverse wake effects of a cavity, a bellow and a pipe junction ( see figure 26) shows that all accelerator components are important and not. only of the rf cavities. 


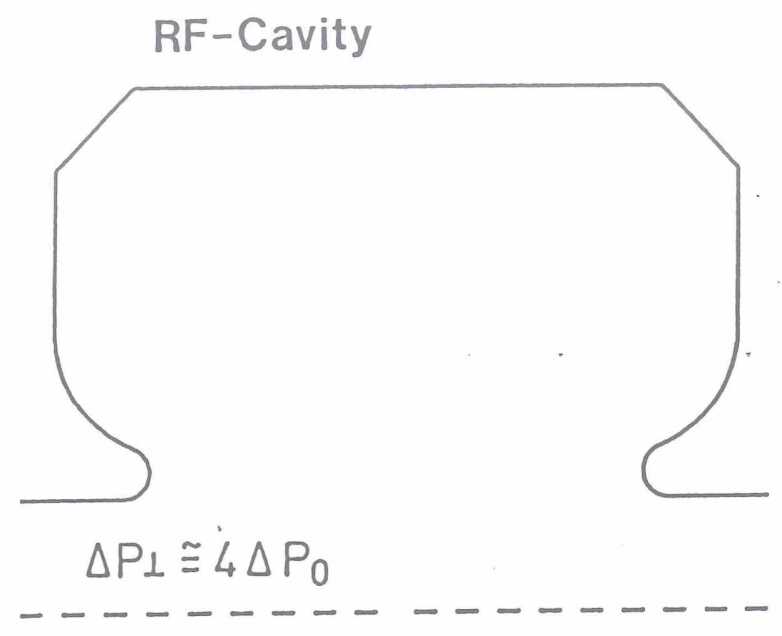

Bellow

$\Delta \mathrm{P}_{\perp} \cong 2.5 \Delta \mathrm{P}_{0}$ กนกนกน

Pipe Junction

$\Delta P_{1} \cong \Delta P_{0} \ldots \ldots$

Figure 26: Comparison of the transverse wake field effects of a cavity, a bellow and a pipe junction. 


\section{Strategies in Accelerator Design}

\subsection{Design Procedure - an Overview}

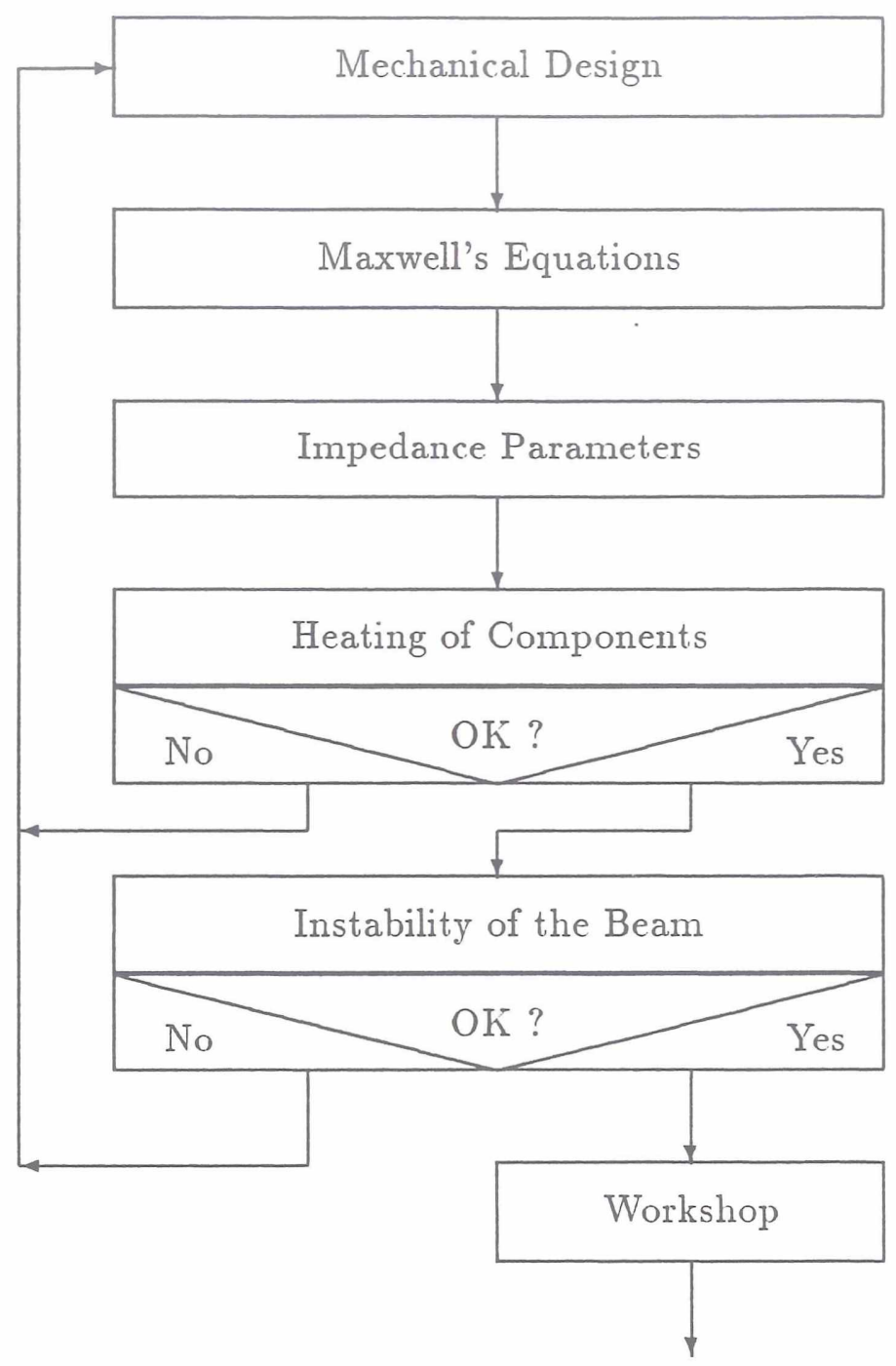

Accelerator that work as predicted

Figure 27: Design procedure for any accelerator component

Since one important reason for current limitations in accelerators are instabilities caused by wake fields all accelerator components should carefully analysed with respect. of their contribution to the impedance. That should be done already at the design stage.

It took over ten years to develop tools to compute impedances very precisely and include this numerical results into instability theory. Today rather sophisticated computer codes are available to calculate the impedance of the various types of accelerator components like cavities, bellows, vacuum chambers etc. Heating and instability ef- 
fects can be evaluated very precisely from this numerical results. One can trust in the computer calculations. It is not necessary to use rather crude madels anymore.

While we can calculate impedances very well, we can not avoid impedances due to accelerating cavities. But by a careful design it is possible to reduce wake field effects significantly.

A good strategy for the design of an accelerator is:

- Minimize parasitic effects of unavoidable components (e.g. cavities).

- Avoidable impedances should be less (equal) than the unavoidable impedances.

- Optimize the overall performance of the accelerator.

In figure 27 an overview of the design procedure for any accelerator component is given. To skip steps of the procedure is certainly a much faster way. But in order to avoid current limitations due to impedances it is always better to go through all steps of the design procedure.

\subsection{Parasitic Losses versus Shunt Impedance}

In this section we will give one example how to minimize parasitic effects of cavities. The accelerating voltage as a function klystron power $P$ and shunt impedance $R$ (we use here the definition from electrical engineering) is given by:

$$
V_{a c c}=\sqrt{2 R P}
$$

In order to save power the shunt impedance can be optimize. Figure 28 shows three examples of typical cavity shapes with different shunt impedances.

The nose cone structure gives the highes shunt impedance. But this type of cavity has also the highes parasitic impedance. While a little klystron power is saved one loose the ability to accelerate higher currents. Even a simple iris loaded structure has a lower parasitic impedance than the noise cone structure. Parasitic effects can be further reduced with the third structure shown in figure 28. This kind of shape is now widely used for superconducting cavities. It is possible to design a single mode cavity based on this kind of shape. In thus a cavity no higher order modes exist. All parasitic effects are due to the continuous part of the impedance. For a quantitative comparison of the various loss parameters we refer to the literature [13]. The conclusion of this section is that it is better to optimize the cavity with respect to parasitic effects than with respect. to the shunt impedance. 

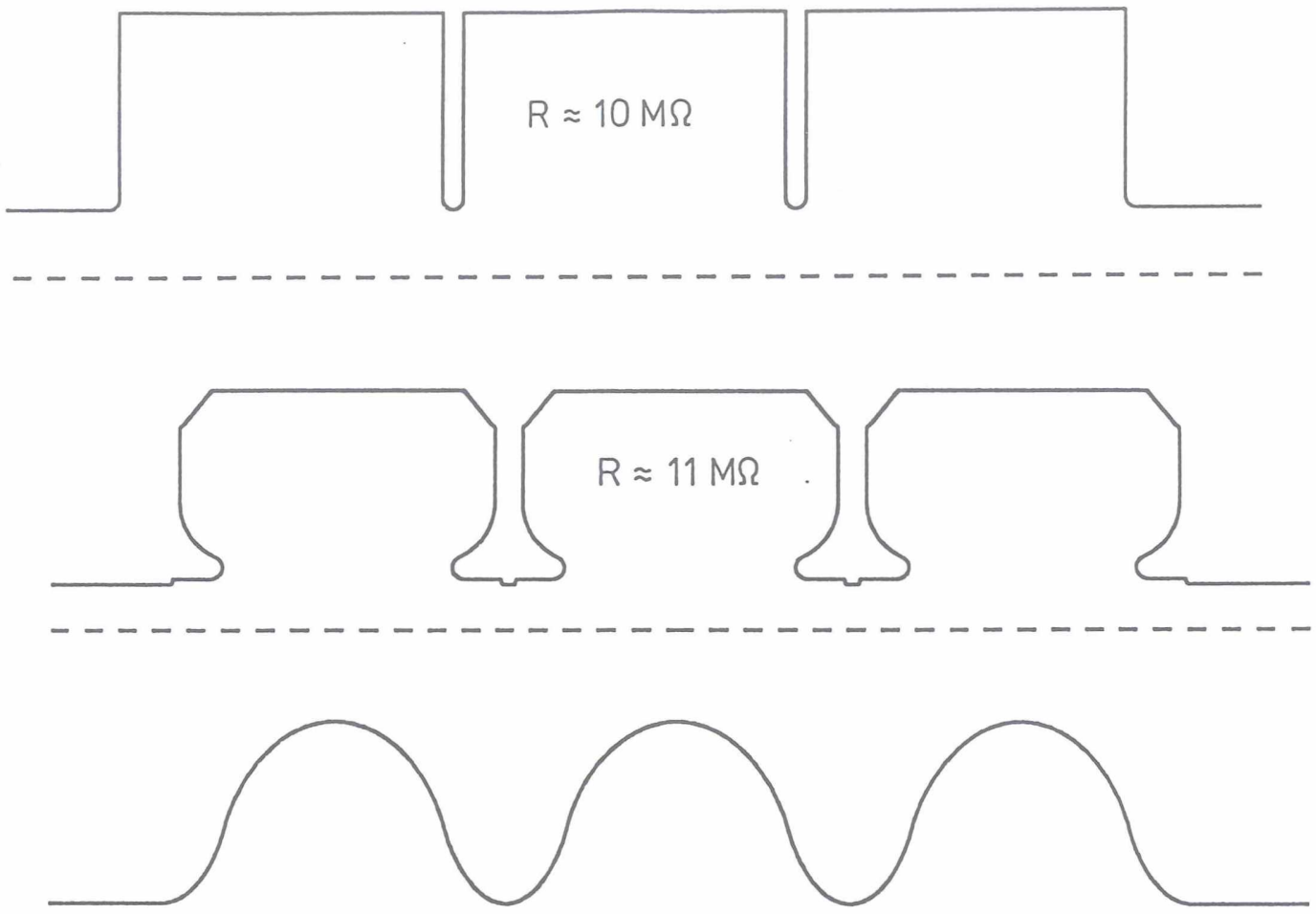

$R \approx 9 M \Omega$

Figure 28: Three differently shaped $500 \mathrm{MHz}$ cavities with their shunt impedances. 


\section{Appendix A: Wake potential and impedance, mode expansion}

The goal of the appendix is to prove that the longitudinal wake potential of a point charge traversing any closed resonator structure along the $z$-axis with the velocity of light can be written as

$$
W_{\|}(s)=\sum_{n} 2 k_{n} \cos \left(\omega_{n} s / c\right)
$$

for all $s>0$. The $k_{n}$ are the loss parameters of the structure. The calculations are firstly worked out in the frequency domain [15]. Then the wake potential is obtained by a Fourier transformation.

The electric and magnetic field can be written in terms of the vector $\boldsymbol{A}$ and the scalar potential $\Phi$.

$$
\boldsymbol{E}=-\frac{\partial}{\partial t} \boldsymbol{A}-\nabla \Phi \quad \boldsymbol{B}=\boldsymbol{\nabla} \times \boldsymbol{A}
$$

The following equations for $\boldsymbol{A}$ and $\Phi$ are equivalent to the the Maxwell equations.

$$
\begin{aligned}
-\nabla^{2} \boldsymbol{A}+\frac{1}{c^{2}} \frac{\partial^{2}}{\partial t^{2}} \boldsymbol{A} & =\mu_{0} \boldsymbol{j}-\frac{1}{c^{2}} \frac{\partial}{\partial t} \nabla \Phi \\
-\nabla^{2} \Phi & =\frac{1}{\epsilon_{0}} \rho .
\end{aligned}
$$

The Coulomb gauge was used for the potentials:

$$
\nabla \cdot \boldsymbol{A}=0 .
$$

The vector potential $\boldsymbol{A}$ can be expanded into the modes $a_{n}$ of the closed resonator structure.

$$
\boldsymbol{A}(\boldsymbol{r}, t)=\sum_{n} q_{n}(t) \boldsymbol{a}_{n}(\boldsymbol{r}) .
$$

The $a_{n}$ have to fulfill the equation

$$
-\nabla^{2} a_{n}(\boldsymbol{r})=\left(\frac{\omega_{n}}{c}\right)^{2} a_{n}(\boldsymbol{r}) .
$$

The $\omega_{n}$ are the eigen frequencies of the structure.

Since the $a_{n}$ form a complete orthogonal system ( $U_{n}$ is a normalization factor)

$$
\frac{\epsilon_{0}}{2} \int d^{3} r \boldsymbol{a}_{n}^{*}(\boldsymbol{r}) \boldsymbol{a}_{m}(\boldsymbol{r})=U_{n} \delta_{n m},
$$

the following relation for the expansion coefficients $q_{n}$ holds:

$$
\left(\omega_{n}^{2} q_{n}(t)+\frac{d^{2}}{d t^{2}} q_{n}(t)\right) U_{n}=\frac{1}{2} \int d^{3} r \boldsymbol{a}_{n}^{*}(\boldsymbol{r}) \boldsymbol{j}(\boldsymbol{r}, t)-\frac{\epsilon_{0}}{2} \frac{\partial}{\partial t} \int d^{3} r \boldsymbol{a}_{n}^{*}(\boldsymbol{r}) \nabla \Phi(\boldsymbol{r}, t) .
$$


For the Coulomb gauge $\nabla a_{n}=0$ we have $a_{n}^{*} \nabla \Phi=\nabla\left(a_{n}^{*} \Phi\right)$. Hence the second integral can be transformed into a surface integral according to Gauss theorem, which vanished due to the boundary condition for $\Phi$.

For the Fourier transform $\widetilde{q_{n}}(\omega)=\int_{-\infty}^{\infty} d t q_{n}(t) \exp (-i \omega t)$ of the expansion coefficient. we obtain:

$$
\widetilde{q_{n}}(\omega)=\frac{1}{2 U_{n}} \frac{-1}{\omega^{2}-\omega_{n}^{2}} \int d^{3} r \boldsymbol{a}_{n}^{*} \tilde{j}(\boldsymbol{r}, \omega) .
$$

The Fourier transform of the vector potential is:

$$
\widetilde{\boldsymbol{A}}(\boldsymbol{r}, \omega)=\sum_{n} \widetilde{q_{n}}(\omega) \boldsymbol{a}_{n}(\boldsymbol{r}) .
$$

The scalar potential can also be expanded into a complete orthogonal system:

$$
\Phi(\boldsymbol{r}, t)=\sum_{n} r_{n}(t) \phi_{n}(\boldsymbol{r})
$$

Let $\phi_{n}$ the eigen vectors of

$$
-\nabla^{2} \phi_{n}(\boldsymbol{r})=\left(\frac{\Omega_{n}}{c}\right)^{2} \phi_{n}(r)
$$

which fulfill the boundary condition $\left[\phi_{n}\right]_{\partial V}=0$.

We choose the $T_{n}$ in such a way that we have:

$$
\frac{\epsilon_{0}}{2}\left(\frac{\Omega_{n}}{c}\right)^{2} \int d^{3} r \phi_{n}^{*}(\boldsymbol{r}) \phi_{m}(\boldsymbol{r})=T_{n} \delta_{n m} .
$$

The expansion coefficients $r_{n}$ for $\Phi$ are given by:

$$
r_{n}(t)=\frac{1}{2 T_{n}} \int d^{3} r \phi_{n}^{*}(\boldsymbol{r}) \rho(\boldsymbol{r}, t) .
$$

Calculation of the impedance

The longitudinal impedance is given by:

$$
Z(x, y, s)=\frac{1}{q} \int_{-\infty}^{\infty} d z \widetilde{E_{z}}(r, \omega) \exp (i \omega z / c) .
$$

The electric field $\widetilde{\boldsymbol{E}}(\boldsymbol{r}, \omega)=i \omega \widetilde{\boldsymbol{A}}(\boldsymbol{r}, \omega)-\nabla \widetilde{\Phi}(\boldsymbol{r}, \omega)$ is excited by a point charge $q$ moving parallel with respect to the $z$-axis.

$$
\begin{aligned}
& \rho(\boldsymbol{r}, t)=q \delta(z-c t) \delta\left(x-x_{0}\right) \delta\left(y-y_{0}\right), \\
& \boldsymbol{j}(\boldsymbol{r}, t)=c \boldsymbol{e}_{z} \rho(\boldsymbol{r}, t) .
\end{aligned}
$$

The Fourier transforms of $\rho$ and $j$ are:

$$
\begin{aligned}
& \tilde{\rho}(r, \omega)=\frac{q}{c} \exp (-i \omega z / c) \delta\left(x-x_{0}\right) \delta\left(y-y_{0}\right), \\
& \tilde{j}_{z}(r, \omega)=q \exp (-i \omega z / c) \delta\left(x-x_{0}\right) \delta\left(y-y_{0}\right) .
\end{aligned}
$$


The Fourier transform of the electric field is given by:

$$
\begin{aligned}
\widetilde{\boldsymbol{E}}(\boldsymbol{r}, \omega)= & q \sum_{n} \frac{-i \omega}{\omega^{2}-\omega_{n}{ }^{2}} \boldsymbol{a}_{n}(\boldsymbol{r}) \frac{1}{2 U_{n}} \int_{-\infty}^{\infty} d \dot{z} a_{n z}^{*}\left(x_{0}, y_{0}, \dot{z}\right) \exp (-i \omega \dot{z} / c) \\
& -\frac{q}{c} \sum_{n} \nabla \phi_{n}(\boldsymbol{r}) \frac{1}{2 T_{n}} \int_{-\infty}^{\infty} d \dot{z} \phi_{n}^{*}\left(x_{0}, y_{0}, \dot{z}\right) \exp (-i \omega \dot{z} / c) .
\end{aligned}
$$

We define the complex voltages:

$$
\begin{aligned}
& V_{n}(x, y, \omega)=\int_{-\infty}^{\infty} d z a_{z n}(x, y, z) \exp (i \omega z / c) \\
& v_{n}(x, y, \omega)=\int_{-\infty}^{\infty} d z\left(\frac{\partial}{\partial z} \phi_{n}\right)(x, y, z) \exp (i \omega z / c) .
\end{aligned}
$$

Now the longitudinal impedance can be calculated:

$$
\begin{aligned}
Z_{0}(x, y, \omega)= & \sum_{n} \frac{-i \omega}{\omega^{2}-\omega_{n}^{2}} \frac{1}{2 U_{n}} V_{n}(x, y, \omega) V_{n}^{*}\left(x_{0}, y_{0}, \omega\right) \\
& +\sum_{n} \frac{i}{\omega} \frac{1}{2 T_{n}} v_{n}(x, y, \omega) v_{n}^{*}\left(x_{0}, y_{0}, \omega\right) .
\end{aligned}
$$

Since $\phi_{n}$ vanished at the boundaries of the structure the following relations could be used:

$$
\frac{1}{c} \int_{-\infty}^{\infty} d z \phi_{n}^{*}\left(x_{0}, y_{0}, z\right) \exp (-i \omega z / c)=\frac{-i}{\omega} \int_{-\infty}^{\infty} d z\left(\frac{\partial}{\partial z} \phi_{n}^{*}\right)\left(x_{0}, y_{0}, z\right) \exp (-i \omega z / c) .
$$

The longitudinal wake potential

is obtained by a Fourier transformation of the impedance:

$$
W_{\|}(x, y, s)=\frac{1}{2 \pi} \int_{C} d \omega Z(x, y, \omega) \exp (i \omega s / c)
$$

Due to causality we have to choose the retarded solution. The contour $C$ is shown in the following figure. For $s>0$ we can close the integration path in the upper half plane and for $s<0$ in the lower half plane. The second term of the impedance, which is due to the scalar potential $\Phi$, does not contribute to the wake potential. Hence this term can be dropped a posteriori for the impedance. This term has a pole for $\omega=0$. Using the Chauchy's theorem we have to calculate the voltage $v_{n}(x, y, \omega)$ for $\omega=0$. But due to the boundary condition the voltage is zero:

$$
v_{n}(x, y, 0)=\int_{-\infty}^{\infty} d z\left(\frac{\partial}{\partial z} \phi_{n}\right)(x, y, z)=\left[\phi_{n}(x, y, z)\right]_{\text {boundary }}=0 .
$$

Hence we have for $s>0$ :

$$
\begin{aligned}
W_{\|}(x, y, s)=\sum_{n} \frac{1}{4 U_{n}} & \left(V_{n}\left(x, y, \omega_{n}\right) V_{n}^{*}\left(x_{0}, y_{0}, \omega_{n}\right) \exp \left(i \omega_{n} s / c\right)\right. \\
& \left.+V_{n}\left(x, y,-\omega_{n}\right) V_{n}^{*}\left(x_{0}, y_{0},-\omega_{n}\right) \exp \left(-i \omega_{n} s / c\right)\right) .
\end{aligned}
$$



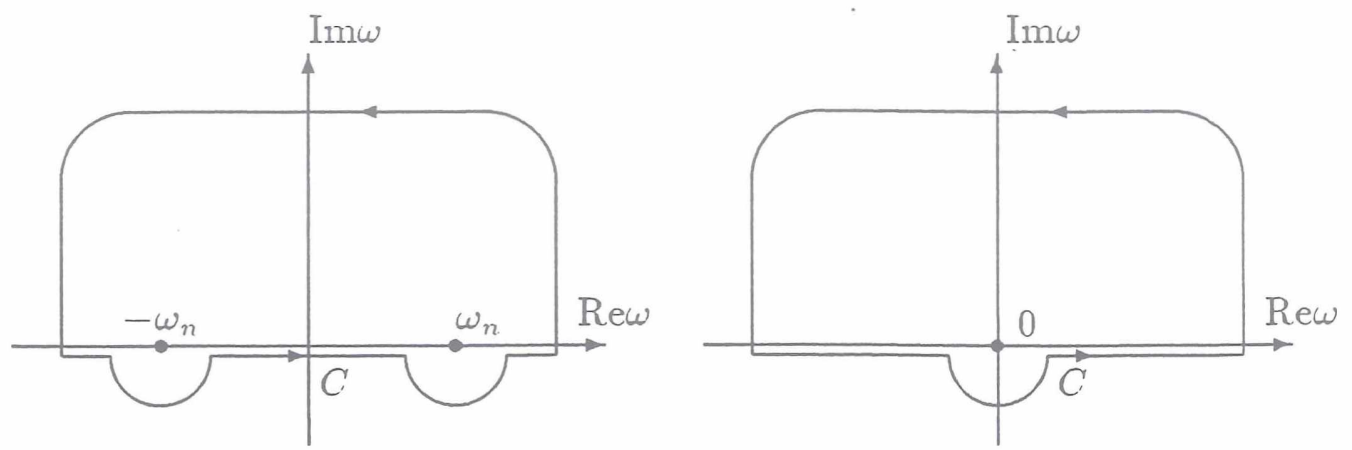

Figure 29: The contour $C$ of the. Fourier integral

Since all eigen vectors $a_{n}$ can be chosen as completely real vectors the following relation holds:

$$
V_{n}^{*}(x, y,-\omega)=V_{n}(x, y, \omega) .
$$

For that choice of eigen vectors one obtains $(s>0)$ :

$$
W_{\|}(x, y, s)=\sum_{n} \frac{1}{4 U_{n}} 2 \operatorname{Re}\left(V_{n}\left(x, y, \omega_{n}\right) V_{n}^{*}\left(x_{0}, y_{0}, \omega_{n}\right) \exp \left(i \omega_{n} s / c\right)\right) .
$$

For the special case $x=x_{0}, y=y_{0}$ the wake potential is given by:

$$
W_{\|}(x, y, s)=\sum_{n} 2 \frac{\left|V_{n}\left(x, y, \omega_{n}\right)\right|^{2}}{4 U_{n}} \cos \left(\omega_{n} s / c\right) .
$$

$\left|V_{n}\left(x, y, \omega_{n}\right)\right|^{2} /\left(4 U_{n}\right)$ is the loss parameter $k_{n}$ of mode number $n$.

For $s=0$ we have:

$$
W_{0}(x, y, 0)=\frac{1}{2 \pi} \int_{C} d \omega Z_{0}(x, y, \omega) .
$$

Since $Z_{0}(x, y, \omega)=-Z_{0}(x, y,-\omega)$ the integral has to be calculated for the two contours $C_{1}$ and $C_{2}$ with radius $\varepsilon$ (see figure 6.2 ).

In the limit $\varepsilon \rightarrow 0$ we obtain for $C_{1}$.

$$
\begin{aligned}
\frac{1}{2 \pi} \int_{C_{1}} d \omega \frac{-i \omega}{\omega^{2}-\omega_{n}^{2}} \frac{\left|V_{n}(x, y, \omega)\right|^{2}}{2 U_{n}} & =\frac{1}{2} \frac{1}{i \pi} \int_{C_{1}} d \omega \frac{1}{\omega+\omega_{n}} \frac{\left|V_{n}(x, y, \omega)\right|^{2}}{4 U_{n}} \\
& =\frac{1}{2} \frac{1}{i \pi} \int_{\pi}^{2 \pi} d \varphi i \frac{\left|V_{n}\left(x, y, \omega_{n}-\varepsilon \epsilon^{i \varphi}\right)\right|^{2}}{4 U_{n}} \\
& \rightarrow \frac{1}{2} k_{n} .
\end{aligned}
$$

The same result is obtained for the contour $C_{2}$. 
The complete result can be written as:

$$
\begin{gathered}
W_{\|}(s)=\theta(s) \sum_{n} 2 k_{n} \cos \left(\omega_{n} s / c\right), \\
\theta(s)= \begin{cases}0 & s<0 \\
1 / 2 & s=0 \\
1 & s>0\end{cases}
\end{gathered}
$$

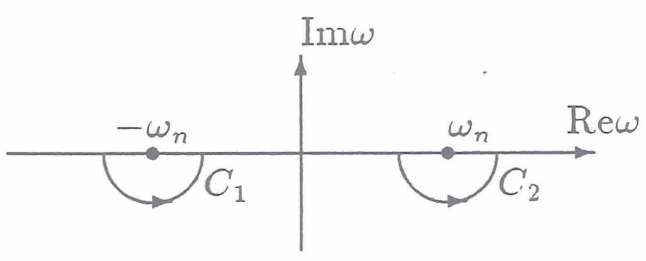

Figure 30: Contours $C_{1}$ and $C_{2}$ 


\section{References}

[1] J.D. Jackson: Classical Electrodynamics 2nd edition Wiley, New York 1975

[2] K.Steinigke:Calculation of the propagation Characteristics of a Circular Waveguide Excentrically Loaded with a Dielectric Rod, Frequenz 44 (1990) 1

[3] A.Piwinski, Longitudinal and transverse wake fields in flat vacuum chambers DESY 84/097, Oct. 1984

[4] W.K.H. Panofsky, W.A. Wenzel, Some Considerations Concerning the Transverse Deflection of Charged Particles in Radio - Frequency Fields, Rev. Sci. Instrum. 27 (1956), 947

[5] K.L.F. Bane, P.B. Wilson, T. Weiland, Wake Fields and Wake Field Acceleration in Physics of High Energy Particle Accelerators (BNL/SUNNY Summer School, 1983) AIP Conf.Proc. No 127 (1984), 876 ed.by M. Month et al.

[6] K.S.Yee:Numerical Solution of Initial Boundary Value Problems Involving Maxwell's Equations in Isotropic Media. IEEE, AP-14, 1966, pp.302-307.

[7] T.Weiland: A Discretization Method for the Solution of Maxuell's Equations for 6 Component Fields. AEÜ, Electron. and Commun., 31(1977),pp.116-120

[8] T.Weiland: On the Numerical Solution of Maxwell's Equations and Applications in the Field of Accelerator Physics. Particle Accelerators 15 (1984), pp.245-292 and references therein

[9] T.Weiland: On the Unique Solution of Maxwellian Eigenvalue Problems in Three Dimensions. Particle Accelerators 17 (1985), pp.227-242

[10] T.Weiland: Transient Electromagnetic Fields Excited by Bunches of Charged Particles in Cavities of Arbitrary Shape. Proceedings of th X1-th International Conference on High Energy Accelerators, Geneva 1980, Birkhauser Verlag, Basel pp 570.

[11] MAFIA code group: Michael Bartsch, Micha Dehler, Martin Dohlus, Frank Ebeling, Peter Hahne, Reinhard Klatt, Frank Krawczyk, Zhang Min, Thomas Pröpper, Dietmar Schmitt, Petra. Schütt, Thomas Weiland, Heike Wolter

Technische Hochschule Darmstadt, Fachbereich 18, Fachgebiet Theorie Elektromagnetischer Felder, Schloßgartenstr. 8, D-6100 Darmstadt, Germany

Michaela Marx, Susan G.Wipf, Deutsches Elektronen-Synchrotron DESY, Notkestr. 85, D-2000 Hamburg 52, Germany

Bernhard Steffen, Kernforschungsanlage Jülich KFA, D-5170 Jülich, Germany 
[12] T. Weiland, On the quantitative prediction of bunch lengthening in high energy electron storage rings DESY 81-088 (1981)

[13] R. Klatt T. Weiland, Comparison of six accelerating cavities with respect to collective effects DEST M-84-06 (1984)

[14] T. Weiland,B. Zotter Wake Potentials of a relativistic current in a cavity Part. Acc. 11,143-151 (1981)

[15] R. Wanzenberg: Longitudinale Strahldynamik im Resonanten Wake Field Beschleuniger Thesis, Universität Hamburg 1990, and DESY M-90-08 Aug. 1990

\section{Acknowledgement}

We would like to thank M. Dohlus who wrote an article for the Mafia code group from which the section about the grid Maxwell equations has been adopted. 